\title{
A close look at the dwarf AGN of NGC 4395: optical and near-IR integral field spectroscopy
}

\author{
Carine Brum, ${ }^{1 \star}$ Marlon R. Diniz ${ }^{\odot},{ }^{1}$ Rogemar A. Riffel ${ }^{\oplus},{ }^{1}$ \\ Alberto Rodríguez-Ardila, ${ }^{2,3}$ Luis C. Ho, ${ }^{4,5}$ Rogério Riffel ${ }^{\odot},{ }^{6}$ Rachel Mason, ${ }^{7,8}$ \\ Lucimara Martins, ${ }^{9}$ Andreea Petric ${ }^{10,7}$ and Rubén Sánchez-Janssen ${ }^{\oplus 11}$ \\ ${ }^{1}$ Departamento de Física, Universidade Federal de Santa Maria, CCNE, 97105-900 Santa Maria, RS, Brazil \\ ${ }^{2}$ Laboratório Nacional de Astrofísica - Rua dos Estados Unidos 154, Bairro das Nações, CEP 37504-364 Itajubá, MG, Brazil \\ ${ }^{3}$ Instituto de Astrofísica de Canarias, C/Vía Láctea s/n, E-38205 La Laguna, Tenerife, Spain \\ ${ }^{4}$ Kavli Institute for Astronomy and Astrophysics, Peking University, Beijing 100871, People's Republic of China \\ ${ }^{5}$ Department of Astronomy, School of Physics, Peking University, Beijing 100871, People's Republic of China \\ ${ }^{6}$ Instituto de Física, Universidade Federal do Rio Grande do Sul, CP 15051, Porto Alegre 91501-970, RS, Brazil \\ ${ }^{7}$ Gemini Observatory, Northern Operations Center, 670 North A'ohoku Place, Hilo, HI 96720, USA \\ ${ }^{8}$ Department of Plant \& Soil Science, University of Vermont, Burlington, VT 05405, USA \\ ${ }^{9}$ NAT-Universidade Cruzeiro do Sul, Rua Galvão Bueno, 868, 01506-000 São Paulo, SP, Brazil \\ ${ }^{10}$ Canada-France-Hawaii Telescope, 65-1238 Mamalahoa Highway, Kamuela, HI 96743, USA \\ ${ }^{11}$ STFC UK Astronomy Technology Centre, Royal Observatory, Blackford Hill, Edinburgh EH9 3HJ, UK
}

Accepted 2019 March 22. Received 2019 March 18; in original form 2018 August 8

\begin{abstract}
Intermediate-mass black holes $\left(10^{3}-10^{5} \mathrm{M}_{\odot}\right)$ in the centre of dwarf galaxies are believed to be analogous to growing active galactic nuclei (AGNs) in the early Universe. Their characterization can provide insight about the early galaxies. We present optical and nearinfrared integral field spectroscopy of the inner $\sim 50 \mathrm{pc}$ of the dwarf galaxy NGC 4395 , known to harbour an AGN. NGC 4395 is an ideal candidate to investigate the nature of dwarf AGN, as it is nearby $(d \approx 4.4 \mathrm{Mpc})$ enough to allow a close look at its nucleus. The optical data were obtained with the Gemini GMOS-IFU covering the $4500-7300 \AA$ spectral range at a spatial resolution of $10 \mathrm{pc}$. The $J$ and $K$-band spectra were obtained with the Gemini NIFS at spatial resolutions of $\sim 5 \mathrm{pc}$. The gas kinematics show a compact, rotation disc component with a projected velocity amplitude of $25 \mathrm{~km} \mathrm{~s}^{-1}$. We estimate a mass of $7.7 \times 10^{5} \mathrm{M}_{\odot}$ inside a radius of $10 \mathrm{pc}$. From the $\mathrm{H} \alpha$ broad-line component, we estimate the AGN bolometric luminosity as $L_{\text {bol }}=(9.9 \pm 1.4) \times 10^{40} \mathrm{erg} \mathrm{s}^{-1}$ and a mass $M_{\mathrm{BH}}=\left(2.5_{-0.8}^{+1.0}\right) \times 10^{5} \mathrm{M}_{\odot}$ for the central black hole. The mean surface mass densities for the ionized and molecular gas are in the ranges (1-2) $\mathrm{M}_{\odot} \mathrm{pc}^{-2}$ and (1-4) $\times 10^{-3} \mathrm{M}_{\odot} \mathrm{pc}^{-2}$ and the average ratio between ionized and hot molecular gas masses is $\sim 500$. The emission-line flux distributions reveal an elongated structure at $24 \mathrm{pc}$ west of the nucleus, which is blueshifted relative to the systemic velocity of the galaxy by $\approx 30 \mathrm{~km} \mathrm{~s}^{-1}$. We speculate that this structure is originated by the accretion of a gas-rich small satellite or by a low-metallicity cosmic cloud.
\end{abstract}

Key words: galaxies: active-galaxies: dwarf-galaxies: individual (NGC 4395)-galaxies: kinematics and dynamics - galaxies: Seyfert.

\section{INTRODUCTION}

One of the most notable astrophysical discoveries of the past three decades is that supermassive black holes (SMBHs) with masses of $M_{\mathrm{BH}} \sim 10^{7}-10^{9} \mathrm{M}_{\odot}$ are common - maybe ubiquitous

^E-mail: carinefisica@gmail.com
- at the centre of massive galaxies (e.g. Kormendy \& Ho 2013). Certain fundamental questions about SMBHs, however, remain to be answered: how were the 'seeds' of black holes formed? How have SMBHs grown over cosmic time? Have they influenced the evolution of their host galaxies, and if so, how? Massive galaxies are thought to have grown through a succession of mergers with other galaxies, and efficient accretion of large amounts of mergersupplied gas accounts for the bulk of the black hole growth in the 
Universe. These processes, however, rapidly obscure the formation history of these SMBHs. They may not represent the initial growth of an $\mathrm{SMBH}$ from its $10^{3}-10^{5} \mathrm{M}_{\odot}$ seed, erasing the effects of a small nascent active galactic nucleus (AGN) on its surroundings.

Simulations suggest that radiative feedback from primordial 'miniquasars' shapes the star formation around them and strongly suppresses their subsequent growth (Jeon et al. 2012). Such processes are impossible to study in high-redshift objects with current facilities. AGNs in local dwarf galaxies, though, offer an opportunity to study relatively small SMBHs $\left(\sim 10^{5}-10^{6} \mathrm{M}_{\odot}\right)$ that may be growing in a way that is analogous to the first kind of such objects. Despite this utility, the first significant numbers of AGN-hosting dwarf galaxies have only recently been discovered (Greene \& Ho 2004, 2007; Dong et al. 2012; Reines, Greene \& Geha 2013), as the community has concentrated its efforts on massive galaxies and the galaxy-SMBH co-evolution suspected on the basis of the well-known $M_{\mathrm{BH}}-\sigma$ relation (e.g. Ferrarese \& Merrit 2000; Gebhardt et al. 2000; Ferrarese \& Ford 2005; Kormendy \& Ho 2013). However the association of intermediate black holes with AGN is still in debate and a recent work by Chilingarian et al. (2018) confirmed the AGN nature of only 10 sources of a sample of 305 candidate galaxies of harbouring intermediate-mass black hole. For example, high-quality integral field spectroscopy (IFS) of He 2-10, a well-known candidate of harbouring an intermediate-mass black hole, show no sign of a central AGN (Cresci et al. 2017).

Near-infrared (near-IR) and optical integral field unit (IFU) observations have been carried out on numerous Seyfert AGN at resolutions of a few $\times 10 \mathrm{pc}$, the spatial scales thought necessary to probe fueling and feedback in low-luminosity objects (Hicks et al. 2013). This has uncovered, for example inflows of molecular (e.g. Riffel et al. 2008; Müller-Sánchez et al. 2009, 2018b; Riffel, Storchi-Bergmann \& Winge 2013; Davies et al. 2014) and ionized (e.g. Schnorr-Müller et al. 2014b) gas and outflows of ionized gas sometimes much larger than the AGN accretion rate (e.g. MüllerSánchez et al. 2011, 2018a; Riffel 2013; Riffel et al. 2013; Lena et al. 2015; Rodríguez-Ardila et al. 2017). Hints of a relation between starburst age and Eddington ratio have also been observed, which suggest that star formation activity predates AGN activation by $\approx 100 \mathrm{Myr}$, and that stellar winds have an influence on AGN fueling (Davies et al. 2007). Rings of intermediate-age stars, consistent with a scenario in which the formation of the stellar ring triggered an episode of nuclear activity are seen for some galaxies (Riffel et al. 2010b, 2011; Diniz et al. 2017), evidence of relatively young nuclear stellar populations and nuclear gas reservoirs in Seyferts that are not detected in quiescent galaxies (Hicks et al. 2013; Mallmann et al. 2018).

As a first step towards understanding the growth of small SMBHs, detailed analysis of the gas kinematics ionization structure and gas morphology must be carried out in dwarfs hosting AGNs. In this context, NGC 4395 is a dwarf spiral galaxy located at a distance $d \approx 4.4 \mathrm{Mpc}(1 \operatorname{arcsec} \approx 20 \mathrm{pc}$ ) (Den Brok et al. 2015), whose broad optical emission lines, X-ray variability, and radio jet unambiguously reveal the presence of an accreting SMBH (Filippenko \& Sargent 1989; Shih, Iwasawa \& Fabian 2003; Wrobel $\&$ Ho 2006). Repetto et al. (2017) using Sloan Digital Sky Survey (SDSS) imaging estimated its stellar mass as $3.82 \times 10^{8} \mathrm{M}_{\odot}$. A striking characteristic of NGC 4395 is that it is a bulgeless spiral galaxy, harbouring a Seyfert 1 nucleus, which defies the correlation between the mass of the central black hole and the mass of the bulge that suggests that the growth of the black hole and the galaxy bulge are coupled. In addition NGC 4395 is one of the lowest mass galaxies with a well-determined dynamical mass for its central black hole (Den Brok et al. 2015). This makes NGC 4395 unique and worthy of a detailed study of its central region.

Reverberation mapping sets the mass of the SMBH of NGC 4395 as $M_{\mathrm{BH}}(3.6 \pm 1.1) \times 10^{5} \mathrm{M}_{\odot}$ (Peterson et al. 2005). A similar value of $M_{\mathrm{BH}}$ is also obtained by modelling the dynamics of the molecular gas in NGC 4395, resulting in $M_{\mathrm{BH}}=4_{-3}^{+8} \times 10^{5} \mathrm{M}_{\odot}$ (Den Brok et al. 2015). An optical continuum image obtained with the Hubble Space Telescope (HST) using the Wide-Field Planetary Camera 2 (WFPC2) and F606W filter reveals an emission structure (hereafter 'blob') at 1".2 of the nucleus (Martini et al. 2003), which has been interpreted as an ionized gas cloud whose line emission lies within the bandwidth of the F606W filter (Den Brok et al. 2015). However, the nature of this blob is still not clear and its study represents a secondary motivation to obtain high-resolution IFS of the central region of NGC 4395, which allows not only mapping the gas emission, but also its kinematics.

This work is organized as follows: Section 2 presents a description of the observations and data reduction procedures. Section 3 shows the emission-line flux distributions, line-ratio maps, velocity fields, and velocity dispersion maps. These maps are interpreted and discussed in Section 4 and the conclusions of this work are presented in Section 5.

\section{OBSERVATIONS AND DATA REDUCTION}

Optical IFS data of NGC 4395 were obtained with the Gemini MultiObject Spectrograph (GMOS) IFU on Gemini North Telescope, covering a spectral range $4500-7300 \AA$. The near-IR data were collected with the Gemini Near-infrared Integral Field Spectrograph (NIFS) at the $J$ and $K$ bands, covering the spectral ranges 1.15 $1.35 \mu \mathrm{m}$ and $2.01-2.43 \mu \mathrm{m}$, for the $J$ - and $K$ band, respectively. The details of the observations and data reduction procedures are discussed in the following sections.

\subsection{The optical IFU data}

NGC 4395 was observed on 2015 June 13 with the GMOS IFU under the program ID: GN-2015A-DD-6 (PI: Mason, R.). We used the one-slit mode, in order to obtain a spectral range that includes both the strongest emission lines in the blue $(\mathrm{H} \beta$ and [O III] $\lambda \lambda 4959,5007)$ and in the red $(\mathrm{H} \alpha,[\mathrm{N} \mathrm{II}] \lambda \lambda 6548,6584$ and [S II] $\lambda \lambda 6716,31)$, commonly observed in active galaxies. For this configuration, the GMOS IFU science field of view (FOV) is $5^{\prime \prime} .0 \times 3$ 3.5. The observations were comprised of four individual exposures of $720 \mathrm{~s}$, resulting a total time of the $48 \mathrm{~min}$ with the spectra centred at $\lambda 5900 \AA$.

Data reduction was performed using the Image Reduction and Analysis Facility (IRAF, Tody 1986, 1993) packages provided by Gemini, using specific tasks developed for GMOS data in the GEMINI.GMOS package and followed the standard procedure of spectroscopic data reduction (see Lena 2014). During the reduction process the bias level was subtracted from each image, followed by flat-fielding and trimming of the spectra. The wavelength calibration was applied to the science data using as reference the spectra of arc lamps, followed by cosmic ray removal and the subtraction of the underlying sky emission. The flux calibration was done by constructing a sensitivity function using the spectra of the standard star EG 131 observed at the same night of the galaxy's observations.

Finally, data cubes for each exposure were constructed with a spatial sampling of $0 . .05 \times 0.0^{\prime \prime} 05$, aligned using the peak of the continuum emission as reference and then median combined to a single cube using the imcombine IRAF task together with a sigma 
clipping algorithm to remove spurious features. The final data cube of NGC 4395 covers the inner 3". $3 \times 4$ ". 95 , after the exclusion of the borders of the FoV, dominated by spurious data. The flux calibration was verified by comparing the spectrum of NGC 4395 obtained by the SDSS (Abolfathi et al. 2018) with the GMOS spectrum, extracted within the same aperture. We found that both spectra are mutually consistent, with flux differences smaller than 5 per cent in most wavelenghts. The highest discrepancy is seen at the red end of the GMOS spectrum, where the fluxes obtained from the GMOS data are about 20 per cent smaller than those obtained from the SDSS spectrum.

The spectral resolution is $1.8 \AA\left(\approx 90 \mathrm{~km} \mathrm{~s}^{-1}\right)$ - derived from the full width at half-maximum (FWHM) of the Ar lamp emission lines used to perform wavelength calibration of the spectra. The angular resolution, obtained from the FWHM of the flux distribution of the broad $\mathrm{H} \alpha$ component, is 0 '. 5 , corresponding to $10 \mathrm{pc}$ at the distance of NGC 4395.

\subsection{The near-IR IFU data}

The Adaptive optics (AO) assisted near-IR $J$ and $K$-band observations of NGC 4395 were obtained with the Near-infrared Integral Field Spectrograph (NIFS; McGregor et al. 2013) on the Gemini North telescope, under the programmes GN-2015A-Q-51 (PI: Mason, R.) and GN-2010A-Q-38 (PI: Seth, A., from the Gemini Observatory Archive), respectively. The total on source exposure time in the $J$ band was $3 \mathrm{~h}$, divided into 10 individual exposures of $480 \mathrm{~s}$ each. In the $K$ band the total exposure time was $1.5 \mathrm{~h}$, divided into 9 exposures of $600 \mathrm{~s}$.

The data reduction for the $J$ - and $K$ band was performed following the same procedures used in previous works (e.g. Riffel et al. 2008; Diniz et al. 2015; Riffel et al. 2017), using specific tasks contained in the NIFS package which is part of GEMINI IRAF package. The reduction process includes the trimming of images, flat-fielding, sky subtraction, wavelength and spatial distortion calibrations. The telluric absorption features were removed using the spectrum of a telluric standard star - of spectral type A, observed at the same nights of the galaxy's observations and the flux calibration was performed by interpolating a blackbody function to the spectrum of the telluric standard star.

For both bands, data cubes for each individual exposure were created at a spatial sampling of $00^{\prime \prime} 05 \times 00^{\prime \prime} 05$, which were then median combined using a sigma clipping algorithm to eliminate the remaining cosmic rays and bad pixels, using the peak of the continuum as reference for astrometry for the distinct cubes. The final data cube for the $J$ band covers the inner 3 .'. $35 \times 3$ 3. 25 region and the final cube for the $K$ band covers the inner 3 .' $4 \times 4$ ". 15 . The FoV of both cubes is larger than the NIFS FoV due to the spatial dithering performed during the observations.

The $J$-band observations were centred at $1.25 \mu \mathrm{m}$. The spectral resolution of the data is $1.8 \AA$, measured from the FWHM of the arc lamp lines, corresponding to a velocity resolution of $43 \mathrm{~km}^{-1}$. The angular resolution is 0.3 , corresponding to $6 \mathrm{pc}$ at the galaxy, as obtained from the FWHM of the flux distribution of the broad component of the $\mathrm{Pa} \beta$ emission line. The $K$-band spectra are centred at $2.2 \mu \mathrm{m}$ and have a spectral resolution of $3.3 \AA\left(45 \mathrm{~km} \mathrm{~s}^{-1}\right)$. The angular resolution of the $K$-band data cube is $0^{\prime \prime} 2$, obtained from the FWHM of the flux distribution of the $\mathrm{Br} \gamma$ broad component emission. This value translates to a physical scale of $4 \mathrm{pc}$ at the galaxy.

In order to verify the flux calibration of the NIFS data, we had extracted nuclear spectra in the $J$ and $K$ band using the same aperture as those of the SDSS spectrum. As there is no spectral overlap between SDSS and NIFS data to allow a direct comparison of the spectra, we have fitted the SDSS spectra by a power law and extrapolated the resulting fit to the $J$ and $K$ bands. We find that the $J$ band fluxes are consistent with the resulting fit, while the $K$-band spectrum shows fluxes 30 per cent smaller than that obtained from the extrapolated fit. Thus, the uncertainty due to the flux calibration is estimated to be up to 30 per cent in the $K$ band and on average smaller than 10 per cent for the $J$ band and optical spectra.

\section{RESULTS}

In the left-hand panel of Fig. 1 we present an $R$-band large-scale image of NGC 4395 obtained with the $0.9 \mathrm{~m}$ telescope of the Kitt Peak National Observatory (KPNO) (Cook et al 2014). Our data cover the very inner region of this image, as indicated by the small rectangle shown in the figure to illustrate the GMOS FoV. The right-hand panels of figure show the optical and nearIR continuum images, obtained from the GMOS and NIFS data cubes, respectively. The optical continuum image was obtained by averaging the fluxes within a spectral window of $200 \AA$ centred at $5700 \AA$. The near-IR ( $K$ band) image was obtained from the NIFS data cube using a spectral window of $100 \AA$ centred at $2.285 \mu \mathrm{m}$. At high flux levels both images show nearly circular flux contours. As the spectra of NGC 4395 do not show any strong stellar absorption, the continuum emission is probably from the AGN. We fitted the nuclear continuum obtained by combining the optical and near-IR spectra shown in Fig. 2 by a power-law function and found a spectral index of 1.1, which is consistent with an AGN origin (e.g. Koski 1978; Kishimoto et al. 2007; Riffel et al. 2009).

In Fig. 2 we show sample spectra of NGC 4395 obtained from the GMOS and NIFS data. These spectra were obtained by integrating the fluxes within circular apertures of 0 '.25 radius centred at the nucleus and at 1.2 west of it - the location of the blob seen in the HST image (Martini et al. 2003). Here, we define the nucleus as being the location of the peak flux in the continuum. The apertures are shown as green circles in the $\mathrm{H} \alpha$ flux map (Fig. 3).

The strongest emission lines are identified in the nuclear spectra. The optical nuclear spectrum of NGC 4395 shows the $\mathrm{H} \alpha, \mathrm{H} \beta$ profiles, [O III] $\lambda \lambda 4959,5007, \mathrm{He} \mathrm{I} \lambda 5876,[\mathrm{O} \mathrm{I}] \lambda \lambda 6300,64$ [N II] $\lambda \lambda 6548,84$, and [S II] $\lambda \lambda 6717,31$ emission lines.

Several emission lines are observed in the near-IR nuclear spectrum of NGC 4395. The most prominent lines are [P II] $\lambda \lambda 1.1471,1.1886$, [Fe II] $\lambda \lambda \lambda 1.2570,1.2946,1.3209$, and $\mathrm{Pa} \beta \lambda 1.2821$ in the $J$ band and $\mathrm{H}_{2} \lambda \lambda 2.1218,2.1542, \mathrm{Br} \gamma \lambda 2.1661$, and $\mathrm{H}_{2} \lambda \lambda 2.2477,2.4084$ in the $K$ band.

The observed emission lines can be used to map the flux distribution, excitation, extinction, and kinematics of the molecular, low- and high-ionization gas. Below, we describe the emission-line profile fitting procedures.

The $2.3 \mu \mathrm{m}$ CO absorption bandheads which are usually prominent in the spectra of nearby galaxies (e.g. Riffel et al. 2015b) were not detected in our NIFS data for NGC 4395. Thus, we were not able to measure the stellar kinematics. These features are expected to be detected on cold and evolved stars and their absence in the spectra of NGC 4395 could be explained if the continuum emission is dominated by a young-stellar population, with the spectra beeing dominated by stars hotter than F0 (see (Reynes, Cushing \& Vacca 2009)). In addtion, we do not see any important absorption feature in the spectra of NGC 4395, thus, a possibility to explain the absence of absorption features is that they can be diluted by the AGN 

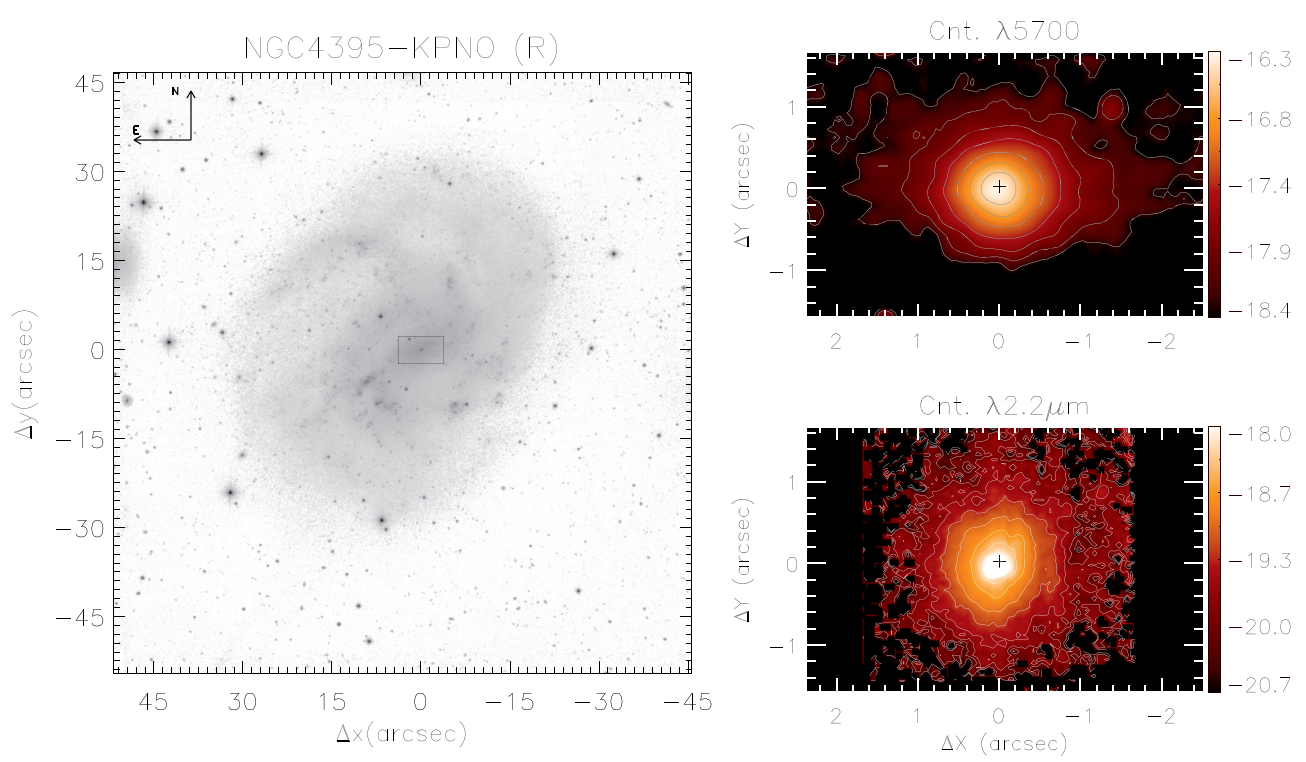

Figure 1. Left-hand panel: large-scale image of NGC 4395 at $R$ band (Cook et al 2014). Right-hand panels: optical (top) and $K$-band (bottom) continuum images obtained from the GMOS and NIFS data cubes, respectively. The colour bars show the fluxes in logarithmic units of erg s $\mathrm{cm}^{-2} \AA^{-1} \mathrm{spaxel}^{-1}$. The central box shown in the left-hand panel corresponds to the GMOS FoV.

continuum, as observed for nearby Seyfert galaxies Riffel et al. (2009).

\subsection{Emission-line profile fitting}

In order to measure the emission-line flux distributions and gas kinematics in the central region of NGC 4395, we fitted the line profiles of $\mathrm{H} \alpha+[\mathrm{N}$ II $] \lambda \lambda$ 6548,6584, [S II] $\lambda \lambda$ 6717,6731, [O III] $\lambda 5007, \mathrm{H} \beta$, $\mathrm{H}_{2} \lambda 2.1218 \mu \mathrm{m}, \mathrm{Pa} \beta$, [Fe II] $\lambda 1.2570 \mu \mathrm{m},[\mathrm{P}$ II] $\lambda 1.8861 \mu \mathrm{m}$, and $\mathrm{Br} \gamma$ at each spaxel over the whole FOV, by Gaussian curves using a modified versions of the PROFIT routine (Riffel 2010c). This routine performs a non-linear least-squares fit of the observed profiles using the MPFIT-FUN routine (Markwardt et al. 2009).

In order to reduce the number of free parameters, we adopted the following constraints: the $[\mathrm{N} \mathrm{II}]+\mathrm{H} \alpha$ emission lines were fitted by keeping tied the centroid velocities and line widths of the [N II] lines and fixing the $[\mathrm{N}$ II $] \lambda 6584 /[\mathrm{N}$ II] $\lambda 6548$ intensity ratio to the theoretical value of 2.98 , given by the ratio of their transition probabilities (Osterbrock \& Ferland 2006). As NGC 4395 has a type 1 AGN, we included in the fit a broad Gaussian component to represent the contribution of the broad-line region (BLR). As this emission is not spatially resolved by our data, the width and centroid velocity of the broad component were kept fixed for all spaxels to the values obtained from the fitting of the nuclear $\mathrm{H} \alpha$ profile shown in Fig. 2, while their amplitudes were allowed to vary to enable for smearing by the seeing. The widths and velocities of the broad components of all recombination lines were constrained to the values measured for the nuclear $\mathrm{H} \alpha$ profile. The choice of the $\mathrm{H} \alpha$ profile is justified as it is the strongest emission line, the broad component is well constrained for the nuclear spectrum and dust extinction seems to not play an important role for the nucleus of NGC 4395. This is indicated by the low $E(B-V)$ values (Fig. 5) and the absence of a dust emission component in the nuclear near-IR spectra (Fig. 2). Similar procedures were successfully adopted in previous works (Brum et al. 2017; Freitas et al. 2018).

The [S II] doublet was fitted by keeping the centroid velocity and line width of the two lines tied, while the $[\mathrm{O} \mathrm{III]} \lambda 5007$,
[Fe II] $\lambda 1.2570 \mu \mathrm{m}$, and $\mathrm{H}_{2} \lambda 2.1218 \mu \mathrm{m}$ were fitted individually with all parameters free.

In all cases, the continuum emission was fitted by a linear regression, as the spectral range of each line fit was small. For each emission line, the fitting routine outputs a data cube with the emission-line fluxes $(F)$, centroid velocity $(V)$, and velocity dispersion $(\sigma)$, as well as their corresponding uncertainties and $\chi^{2}$ maps. These cubes were used to construct the two-dimensional maps presented in the next sections. For the $\mathrm{H}$ recombination lines, all presented maps are based on the narrow-component measurements.

In Table 1 we present the fluxes of optical and near-IR emission lines measured for the nucleus and the blob region using the spectra shown in Fig. 2.

\subsection{Flux distributions}

The flux distributions for the emission lines $\mathrm{Pa} \beta, \mathrm{H} \alpha$, [Fe II $] \lambda 1.2570 \mu \mathrm{m}, \quad[\mathrm{O} \mathrm{III}] \lambda 5007 \AA, \quad \mathrm{H}_{2} \lambda 2.1218 \mu \mathrm{m}, \quad$ and $[\mathrm{N}$ II] $\lambda 6584 \AA$ are presented in Fig. 3. Although other emission lines are observed in the spectra of NGC 4395, we constructed maps for the flux distributions and kinematics only for these emission lines, as they present the highest signal-to-noise $(\mathrm{S} / \mathrm{N})$ ratio among their species. Although the $[\mathrm{S} \mathrm{II}]$ emission lines are detected at most locations of the GMOS FoV, we do not show the corresponding maps, as they are similar to that of [N II] $\lambda 6584 \AA$ line. The colour bars show the flux in logarithmic units of $10^{-17} \mathrm{erg} \mathrm{s}^{-1} \mathrm{~cm}^{-2}$ spaxel $^{-1}$. The gray regions represent masked locations where the uncertainty in the flux is larger than 30 per cent, and we were not able to get good fits of the line profiles due to the low $\mathrm{S} / \mathrm{N}$ ratio or non-detection of the corresponding emission line. The left-hand panels of Fig. 3 present the flux maps for the near-IR lines, while the right-hand panels present the maps for the optical lines. In order to make a comparison between the distinct maps, they are all shown in the same spatial scale.

The flux maps of Fig. 3 show that all emission lines present their intensity peak at the nucleus of the galaxy, and an elongated and curved structure is seen extending to up to 2 arcsec south-west 

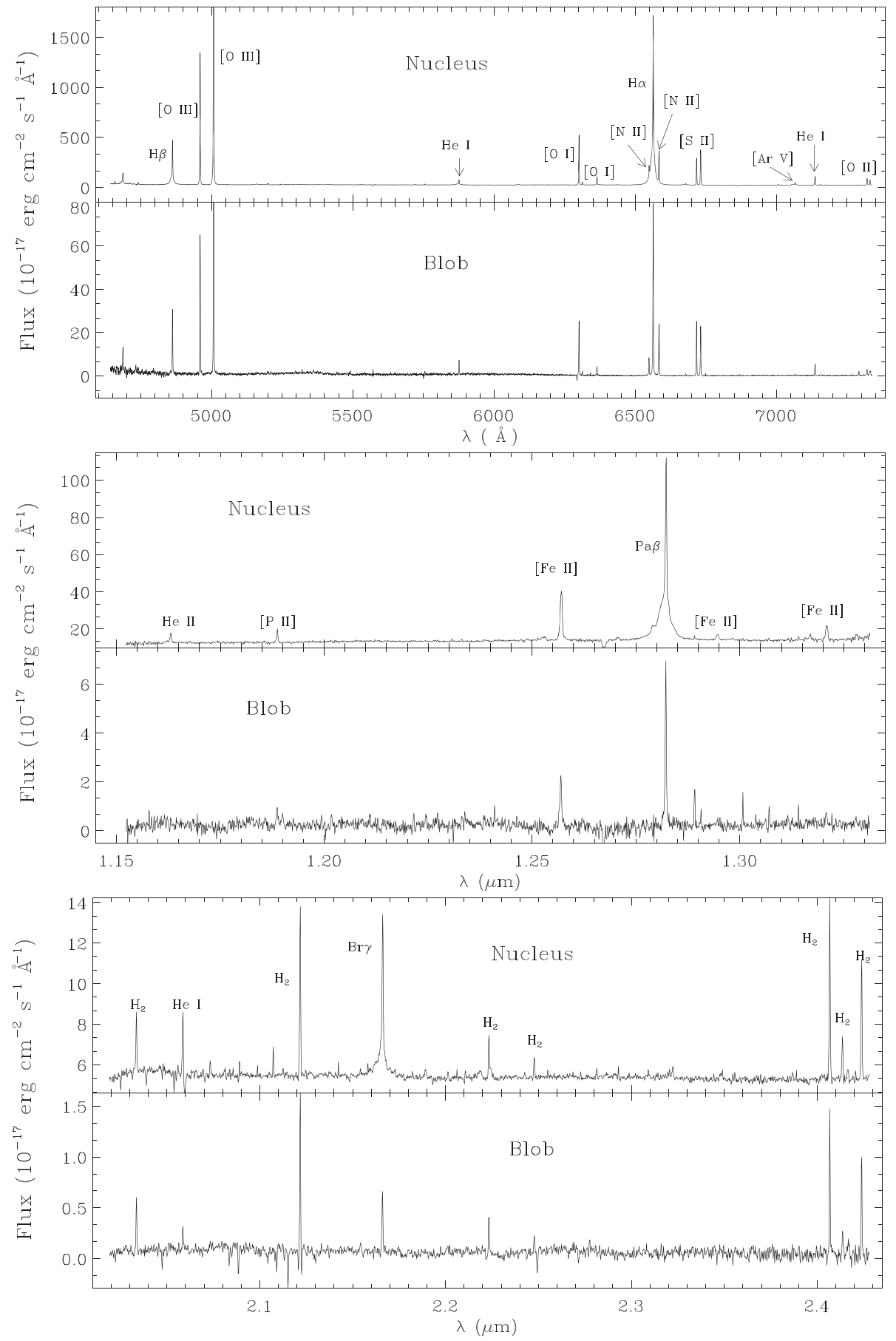

Figure 2. Optical and near-IR spectra of NGC 4395 obtained from the GMOS and NIFS data cubes by integrating the fluxes within a circular aperture of 0 "' 25 radius centred at the nucleus and at 1".2 west (at the blob). The strongest emission lines of each band are identified in the nuclear spectrum. The first two plots show the optical spectra, the third and forth plots show the $J$-band spectra, and the last two show the $K$-band spectra.

from the nucleus. This structure is co-spatial with the blob seen in the HST broad-band image presented by Martini et al. (2003). This correspondence is clearly seen in Fig. 4, where we present the HST image of NGC 4395 obtained through the filter F606W in grey scale and show the flux contours for the $\mathrm{H} \alpha$ flux as green contours. As the angular resolution of the GMOS data is lower than that of NIFS data, the blob seems larger in the flux maps of the optical lines. As our continuum images (right-hand panels of Fig. 1) do not show any enhanced emission to the south-west, the only plausible interpretation is that the blob seen in the HST image is due to contamination of the broad-band continuum by line emission.

The flux maps for $\mathrm{H} \alpha$, [O III], and [N II] are very similar. They show extended emission in almost the entire FOV. The elongated structure to the south-west is clearly observed. The $\mathrm{Pa} \beta$, [Fe II], and $\mathrm{H}_{2}$ lines present a more compact emission compared to the GMOS data, probably due to the higher resolution of the NIR data. 

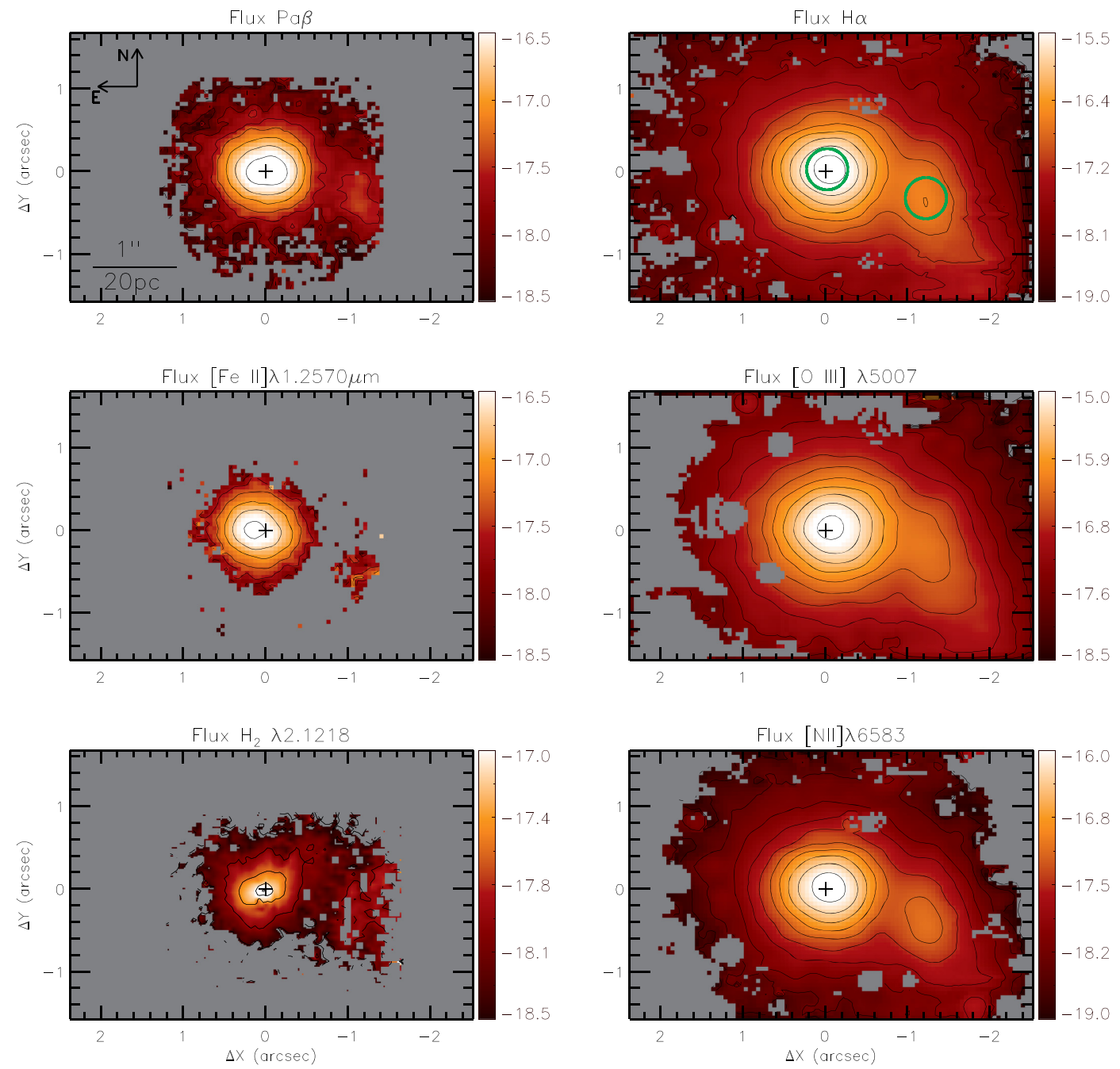

Figure 3. Near-IR (left-hand panels) and optical (right-hand panels) emission-line flux distributions of NGC 4395 . The colour bars show the fluxes in logarithmic units ( $\mathrm{erg} \mathrm{s}^{-1} \mathrm{~cm}^{-2}$ spaxel ${ }^{-1}$ ) and the grey regions correspond to locations where the lines were not detected or good fits were not possible. The emission lines are identified in each panel and the fluxes are not corrected by extinction. The green circles shown in the H $\alpha$ map correspond to the apertures used to extract the spectra shown in Fig. 2.

\subsection{Emission-line ratios}

We have used the emission-line fluxes to construct the intensity-line ratio maps shown in Fig. 5 and Fig. 6 using the optical and near-IR lines, respectively.

\subsubsection{Optical lines}

In the top-left panel of Fig. 5 we show a reddening map, with values of $E(B-V)$ obtained from the $\mathrm{H} \alpha / \mathrm{H} \beta$ line ratio by

$E(B-V)=1.38 \log \frac{\left(\frac{H \alpha}{H \beta}\right)}{2.87}$.

This equation was derived by adopting the Cardelli, Clayton \& Mathis (1989) reddening law and the adopting of the $H \alpha / \mathrm{H} \beta$ theoretical ratio for the Case B assuming a electron temperature of $T_{e}=10000 \mathrm{~K}$ (Osterbrock \& Ferland 2006) for the low-density limit. At some locations the equation above returned values slightly smaller then zero, which were set as zero in the $E(B-V)$ map. The $E(B-V)$ map shows overall small values, with the highest ones of up to 0.3 mag being seen at the nucleus. A small dust extinction is expected for the central region of NGC 4395, as it does not present any signature of dust structures close to the nucleus, as revealed by the $V-H$ colour map presented by Martini et al. (2003).

In the top-right panel of Fig. 5 we show the $[\mathrm{N} \mathrm{II}] / \mathrm{H} \alpha$ flux ratio map. It presents a narrow range of values, between 0.22 and 0.35 . The highest values are seen in the blob region. Other locations show $[\mathrm{N}$ II $] / \mathrm{H} \alpha$ values smaller then 0.3 . The $[\mathrm{O} \mathrm{III}] / \mathrm{H} \beta$ ratio map (bottom-left panel of Fig. 5) shows the highest values of up to 11 at the nucleus, while the smallest values (6-8) are observed at the blob region.

The electron density $\left(N_{\mathrm{e}}\right)$ map is shown in the bottom-right panel of Fig. 5. The $N_{\mathrm{e}}$ at each spaxel was derived from the intensity ratio $[\mathrm{S}$ II $] \lambda 6716 / \lambda 6731$ using the TEMDEN routine of the NEBULAR package of the STSDAS.IRAF package, assuming an electron temperature of the $10000 \mathrm{~K}$. The $N_{\mathrm{e}}$ values of $\approx 1800 \mathrm{~cm}^{-3}$ are observed at the nucleus. In the blob region, typical values are $N_{\mathrm{e}}<500 \mathrm{~cm}^{-3}$.

\subsubsection{Near-IR lines}

The excitation mechanisms of the $[\mathrm{Fe} \mathrm{II}]$ and $\mathrm{H}_{2}$ emission lines can be investigated using emission-line ratio maps. Fig. 6 shows 
Table 1. Optical and near-IR emission-line fluxes for the nucleus and the blob region (1". 2 west), measured within circular aperture of 0 '. 25 using the spectra shown in Fig. 2. The fluxes are not corrected by extinction and are in units of $10^{-17} \mathrm{erg} \mathrm{s}^{-1} \mathrm{~cm}^{-2}$. For recombination lines we labelled the broad and narrow line components as $(\mathrm{B})$ and $(\mathrm{N})$, respectively.

\begin{tabular}{|c|c|c|c|}
\hline$\lambda_{0}(\AA)$ & ID & Nuc & Blob \\
\hline 4658 & {$[\mathrm{Fe} \mathrm{III}]$} & $39.3 \pm 1.3$ & - \\
\hline 4685 & He II & $189.3 \pm 3.4$ & $24.1 \pm 2.3$ \\
\hline 4711 & [Ar IV] & $33.1 \pm 2.5$ & - \\
\hline 4740 & [Ar IV] & $38.2 \pm 2.1$ & $5.5 \pm 1.5$ \\
\hline 4862 & $\mathrm{H} \beta(\mathrm{N})$ & $809.4 \pm 4.0$ & $67.1 \pm 1.1$ \\
\hline 4862 & $\mathrm{H} \beta(\mathrm{B})$ & $1152.0 \pm 15.5$ & - \\
\hline 4959 & [O III] & $3137.0 \pm 2.3$ & $138.1 \pm 1.0$ \\
\hline 5007 & [O III] & $9463.0 \pm 2.4$ & $418.6 \pm 0.9$ \\
\hline 5158 & {$[\mathrm{Fe}$ VII $]$} & $46.4 \pm 4.4$ & $2.1 \pm 0.9$ \\
\hline 5199 & {$[\mathrm{Fe} \mathrm{II}]$} & $58.5 \pm 3.0$ & $1.3 \pm 0.3$ \\
\hline 5309 & {$[\mathrm{Ca} \mathrm{V}]$} & $14.9 \pm 2.4$ & - \\
\hline 5316 & {$[\mathrm{Fe} \mathrm{II}]$} & $4.1 \pm 1.1$ & - \\
\hline 5335 & {$[\mathrm{Fe} \mathrm{VI}]$} & $13.8 \pm 2.1$ & - \\
\hline 5518 & [Cl III] & $6.6 \pm 1.8$ & - \\
\hline 5537 & [Cl III] & $7.1 \pm 2.2$ & - \\
\hline 5577 & {$[\mathrm{OI}]$} & $12.1 \pm 1.2$ & - \\
\hline 5720 & {$[\mathrm{Fe}$ VII $]$} & $27.3 \pm 4.1$ & - \\
\hline 5754 & {$[\mathrm{~N}$ II $]$} & $19.8 \pm 1.4$ & $3.9 \pm 0.9$ \\
\hline 5876 & He I(N) & $80.9 \pm 1.2$ & $14.1 \pm 0.8$ \\
\hline 5876 & He I(B) & $172.6 \pm 8.0$ & - \\
\hline 6087 & [Fe VII] & $27.4 \pm 1.4$ & - \\
\hline 6300 & {$[\mathrm{OI}]$} & $1111.0 \pm 1.0$ & $52.6 \pm 1.0$ \\
\hline 6312 & [S III] & $64.3 \pm 1.0$ & $3.9 \pm 0.4$ \\
\hline 6364 & {$[\mathrm{OI}]$} & $183.1 \pm 1.1$ & $8.7 \pm 0.4$ \\
\hline 6548 & {$[\mathrm{~N}$ II $]$} & $320.1 \pm 2.4$ & $17.5 \pm 0.3$ \\
\hline 6563 & $\mathrm{H} \alpha(\mathrm{N})$ & $3308.0 \pm 2.0$ & $182.6 \pm 0.3$ \\
\hline 6563 & $\mathrm{H} \alpha(\mathrm{B})$ & $6143.0 \pm 18.4$ & - \\
\hline 6584 & {$[\mathrm{~N} \mathrm{II}]$} & $868.7 \pm 2.1$ & $52.8 \pm 0.3$ \\
\hline 6677 & $\operatorname{He} \mathrm{I}(\mathrm{N})$ & $22.8 \pm 1.1$ & $1.6 \pm 0.2$ \\
\hline 6677 & He I(B) & $74.6 \pm 7.4$ & - \\
\hline 6717 & [S II] & $659.8 \pm 1.0$ & $56.9 \pm 0.2$ \\
\hline 6731 & [S II] & $887.6 \pm 1.1$ & $54.3 \pm 0.2$ \\
\hline 7005 & {$[\mathrm{ArV}]$} & $27.6 \pm 1.0$ & - \\
\hline 7064 & $\operatorname{He} \mathrm{I}(\mathrm{N})$ & $46.5 \pm 1.0$ & $1.7 \pm 0.3$ \\
\hline 7064 & He I(B) & $110.4 \pm 6.9$ & - \\
\hline 7135 & [Ar III] & $242.6 \pm 1.2$ & $11.7 \pm 0.6$ \\
\hline 7155 & {$[\mathrm{Fe} \mathrm{II}]$} & $35.0 \pm 1.2$ & $2.08 \pm 0.7$ \\
\hline 7172 & {$[\mathrm{Fe}$ II $]$} & $10.9 \pm 1.5$ & - \\
\hline 7319 & [O II] & $211.9 \pm 2.6$ & $7.7 \pm 0.3$ \\
\hline 7329 & [O II $]$ & $182.3 \pm 2.8$ & $9.5 \pm 0.4$ \\
\hline 1.16296 & He II(N) & $25.2 \pm 2.1$ & - \\
\hline 1.16296 & He II(B) & $21.2 \pm 6.9$ & - \\
\hline 1.18861 & {$[\mathrm{P}$ II $]$} & $25.2 \pm 1.6$ & $4.8 \pm 1.0$ \\
\hline 1.25702 & [Fe II $]$ & $176.3 \pm 3.6$ & $11.3 \pm 1.2$ \\
\hline 1.28216 & $\mathrm{~Pa} \beta(\mathrm{N})$ & $261.4 \pm 2.0$ & $22.3 \pm 1.0$ \\
\hline 1.28216 & $\mathrm{~Pa} \beta(\mathrm{B})$ & $754.3 \pm 13.2$ & - \\
\hline 1.29462 & {$[\mathrm{Fe} \mathrm{II}]$} & $20.7 \pm 1.5$ & $0.6 \pm 0.3$ \\
\hline 1.32092 & {$[\mathrm{Fe} \mathrm{II}]$} & $50.1 \pm 4.6$ & $2.5 \pm 0.8$ \\
\hline 2.03376 & $\mathrm{H}_{2}$ & $16.9 \pm 1.6$ & $3.2 \pm 0.5$ \\
\hline 2.05869 & $\operatorname{He} \mathrm{I}(\mathrm{N})$ & $19.8 \pm 2.0$ & $1.2 \pm 0.4$ \\
\hline 2.05869 & He I(B) & $5.2 \pm 1.1$ & - \\
\hline 2.12183 & $\mathrm{H}_{2}$ & $51.0 \pm 1.5$ & $9.2 \pm 0.6$ \\
\hline 2.16612 & $\operatorname{Br} \gamma(\mathrm{N})$ & $45.6 \pm 1.3$ & $3.8 \pm 0.4$ \\
\hline 2.16612 & $\operatorname{Br} \gamma(\mathrm{B})$ & $59.7 \pm 6.4$ & - \\
\hline 2.22344 & $\mathrm{H}_{2}$ & $13.8 \pm 1.1$ & $2.3 \pm 0.4$ \\
\hline 2.24776 & $\mathrm{H}_{2}$ & $6.5 \pm 1.0$ & $1.1 \pm 0.3$ \\
\hline 2.40660 & $\mathrm{H}_{2}$ & $53.9 \pm 1.4$ & $7.2 \pm 0.5$ \\
\hline 2.41367 & $\mathrm{H}_{2}$ & $15.1 \pm 1.6$ & $2.2 \pm 0.8$ \\
\hline 2.42370 & $\mathrm{H}_{2}$ & $38.1 \pm 1.4$ & $2.8 \pm 0.4$ \\
\hline
\end{tabular}

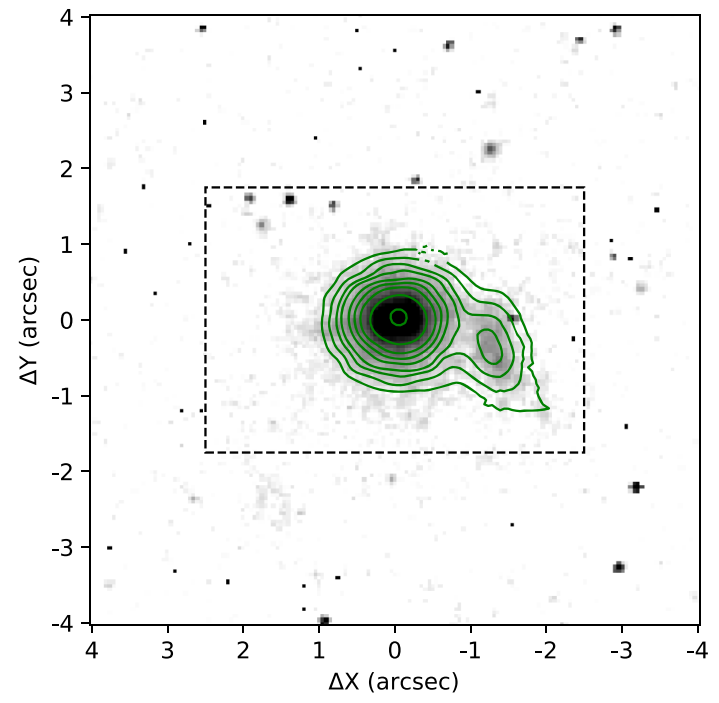

Figure 4. Archival F606W HST image shown in grey scale. The dashed rectangle shows the GMOS IFU FOV. H $\alpha$ flux contours from the GMOS data are shown as green contours.

the [Fe II] $\lambda 1.2570 \mu \mathrm{m} /[\mathrm{P} \mathrm{II}] \lambda 1.8861 \mu \mathrm{m}$ (left-hand panel), [Fe II] $\lambda 1.2570 \mu \mathrm{m} / \mathrm{Pa} \beta$ (middle panel), $\mathrm{H}_{2} \lambda 2.1218 \mu \mathrm{m} / \mathrm{Br} \gamma$ (right-hand panel) line flux ratio maps. These maps are shown only for the inner $3 \operatorname{arcsec} \times 3 \operatorname{arcsec}$. The first two line ratios are useful to investigate the origin of the $[\mathrm{Fe} \mathrm{II}]$ emission and the third map can be used to investigate the $\mathrm{H}_{2}$ emission origin (e.g. Rodríguez-Ardila et al. 2004; Rodríguez-Ardila, Riffel \& Pastoriza 2005; Riffel et al. 2013; Colina et al. 2015; Lamperti et al. 2017).

The $[\mathrm{Fe} \mathrm{II}] /[\mathrm{P} \mathrm{II}]$ map shows values ranging from $\approx 3$ to 12 , with the smallest values observed west of the nucleus at distances smaller than 0 .'8. In the blob region values ranging from 6 to 9 are observed.

The $[\mathrm{Fe} \mathrm{II}] / \mathrm{Pa} \beta$ line ratio shows values ranging from 0.3 to up to 1.0 , with the highest values observed east of the nucleus and the smallest values, observed to the west. In the blob region, high values are observed. The $\mathrm{H}_{2} / \mathrm{Br} \gamma$ line ratio shows values in the range from 0.8 to 3.0 , with the highest values seen at the blob region, while at the nucleus the $\mathrm{H}_{2} / \mathrm{Br} \gamma \approx 1.2$.

\subsection{Velocity and velocity dispersion maps}

In Fig. 7 we present the centroid velocity fields after subtraction of the heliocentric systemic velocity of $325 \mathrm{~km} \mathrm{~s}^{-1}$, which was obtained through a model fitted to the $\mathrm{H} \alpha$ velocity field, as discussed in Section 4.5. The uncertainties in velocity are smaller than $10 \mathrm{~km} \mathrm{~s}^{-1}$ at most locations. The gray regions in the figures represent locations where the $\mathrm{S} / \mathrm{N}$ was not high enough to allow the fitting of the line profiles.

The velocity fields derived for all emission lines are similar, presenting blueshifts of up to $30 \mathrm{~km} \mathrm{~s}^{-1}$ to the east side of the nucleus and similar redshifts to the west at distances smaller than 1 arcsec from the nucleus. In addition, blueshifts of up to $30 \mathrm{~km} \mathrm{~s}^{-1}$ are observed west of the nucleus, co-spatial with the blob region.

Fig. 8 shows the velocity dispersion maps $(\sigma)$, corrected for the instrumental broadening. The uncertainties in the velocity dispersion maps are smaller than $10 \mathrm{~km} \mathrm{~s}^{-1}$ at most locations. All maps show higher $\sigma$ values east of the nucleus and smaller values are observed to the west. The $\mathrm{Pa} \beta, \mathrm{H} \alpha$, and [N II] $\sigma$ maps show the 

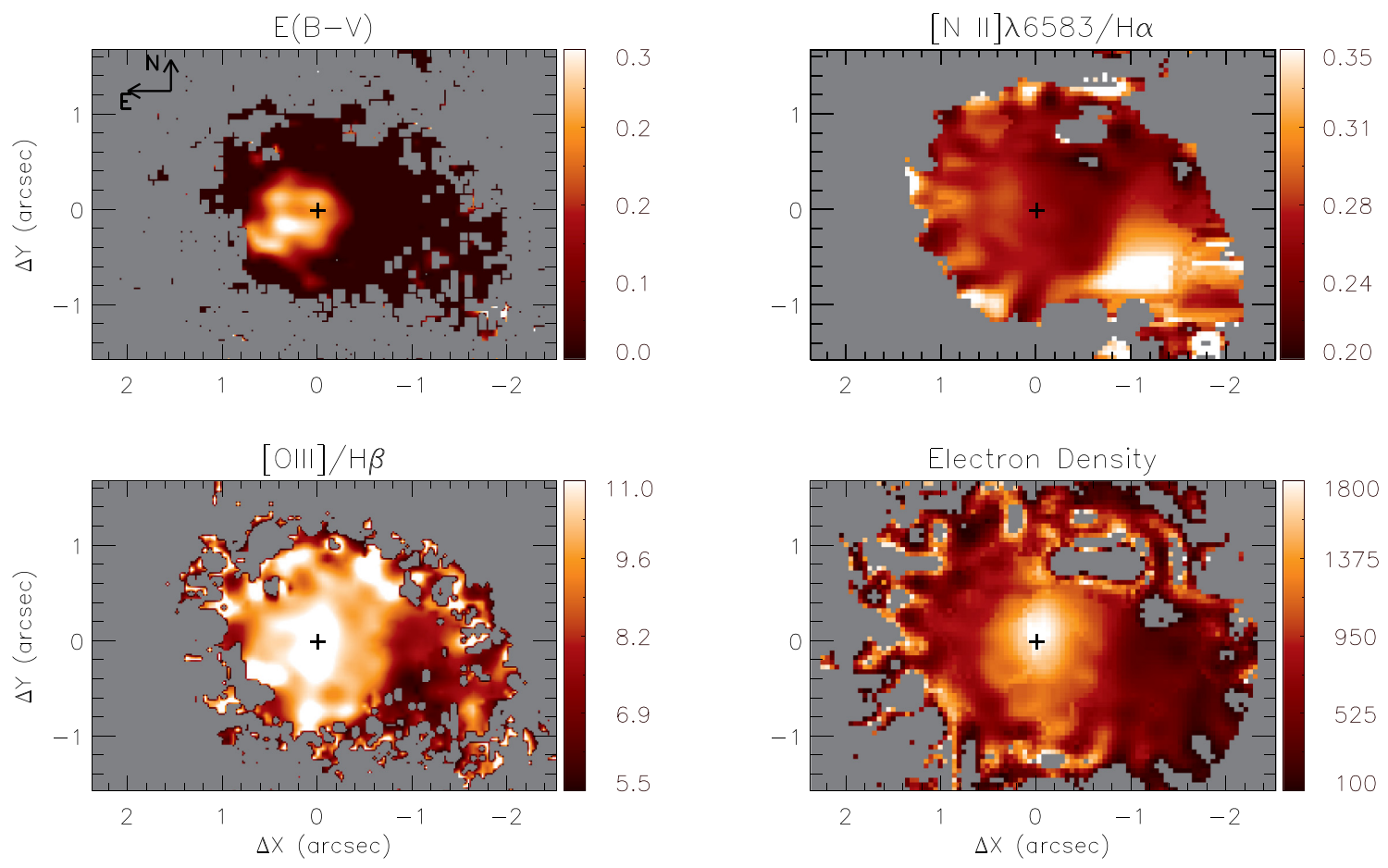

Figure 5. Emission-line ratio maps from the GMOS data. Top-left panel: $E(B-V)$ map obtained from the $\mathrm{H} \alpha / \mathrm{H} \beta$ flux ratio. Top-right: [N II] $] 6584 / \mathrm{H} \alpha$ map. Bottom-left: [O III] $] \lambda 5007 / \mathrm{H} \alpha$ map. Bottom-right: electron density map obtained from the [S II] lines. Density units are $\mathrm{cm}^{-3}$. The gray regions correspond to masked locations due to the low $\mathrm{S} / \mathrm{N}$ of one or both lines of the ratio.
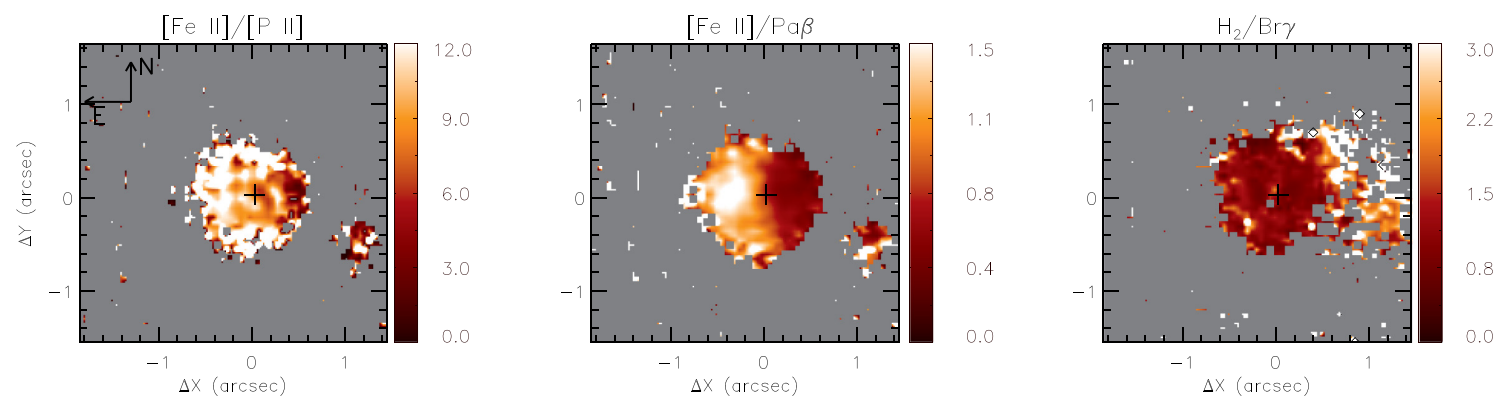

Figure 6. Emission-line ratio maps from the NIFS data. From left to right, the $[\mathrm{Fe} I \mathrm{II}] \lambda 1.2570 \mu \mathrm{m} /[\mathrm{P}$ II $] \lambda 1.8861 \mu \mathrm{m},[\mathrm{Fe} I \mathrm{I}] \lambda 1.25704 \mu \mathrm{m} / \mathrm{Pa} \beta$, and $\mathrm{H}_{2} \lambda 2.1218 \mu \mathrm{m} / \mathrm{Br} \gamma$ ratio maps are shown.

highest values of up to $60 \mathrm{~km} \mathrm{~s}^{-1}$ to the east of the nucleus and lower values, down to $40 \mathrm{~km} \mathrm{~s}^{-1}$, are seen to the west. The mean $\sigma$ value at the blob region is about $55 \mathrm{~km} \mathrm{~s}^{-1}$. A similar behaviour is observed in the $[\mathrm{Fe}$ II $]$ and $[\mathrm{O}$ III] $\sigma$ maps, but with overall higher $\sigma$ values. The smallest $\sigma$ values are observed in the $\mathrm{H}_{2} \sigma$ map, which shows $\sigma<$ $30 \mathrm{~km} \mathrm{~s}^{-1}$ to the west and $30<\sigma<40 \mathrm{~km} \mathrm{~s}^{-1}$ are observed east of the nucleus. Previous studies indicate that the velocity dispersion of the gas is similar to that of the stars (Greene \& Ho 2005a; Ho 2009; Kong \& Ho 2018). The stellar velocity dispersion for the nucleus of NGC $4395\left(\sigma_{\star} \approx 30 \mathrm{~km} \mathrm{~s}^{-1}\right)$ was published by Filippenko \& Ho (2003), measured from the Ca II absorption features. This value is similar to that observed for the $\mathrm{H}_{2}$ and somewhat smaller than the $\sigma$ values for the ionized gas.

In general the $\mathrm{H}_{2}$ presents the smallest $\sigma$ values, followed by the [N II] and $\mathrm{H}$ recombination lines and the highest $\sigma$ values are seen for the highest ionization lines of [Fe II] and [O III].

\section{DISCUSSION}

\subsection{Gas distributions}

The emission-line flux distributions (Fig. 3) reveal the presence of an elongated to the south-west, co-spatial with a blob seen in HST broad-band images (Martini et al. 2003; Den Brok et al. 2015). Due to the higher angular resolution, this structure is better resolved in the near-IR emission-line flux maps, while for the optical lines it appears as an elongated and curved structure. Considering that the our continuum images (Fig. 1) do not show any enhanced emission at the blob location, the most plausible interpretation is that the blob seen in the HST image is due to line emission of ionized gas within the bandwidth of the F606W HST filter. This scenario was already suggested by Den Brok et al. (2015) and in Section 4.5 we speculate on the origin of this blob. 

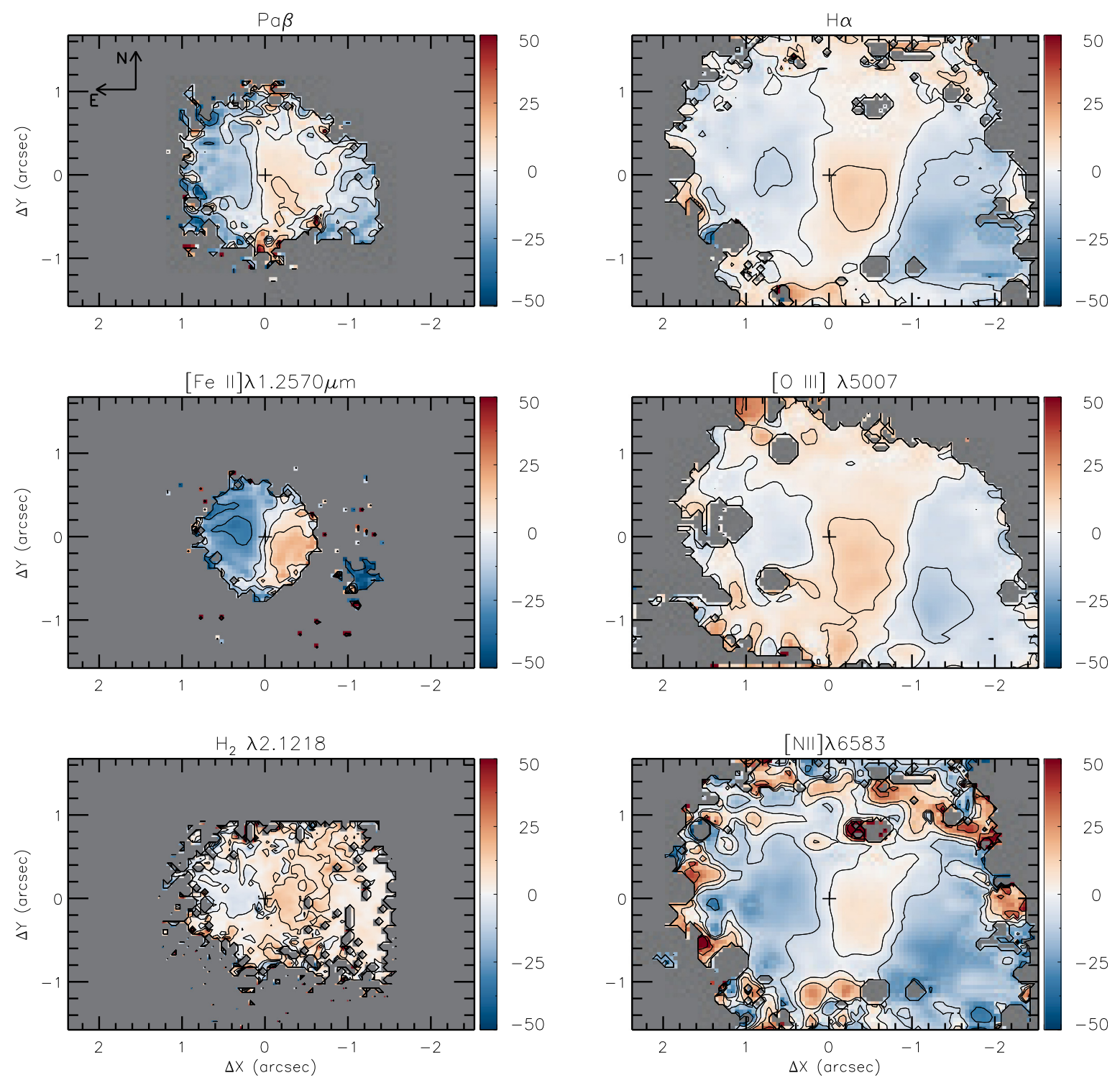

Figure 7. Centroid velocity fields for the $\mathrm{Pa} \beta, \mathrm{H} \alpha$, [Fe II], [O III], $\mathrm{H}_{2}$, and [N II] emission lines. The colour bar shows the velocities in units of km $\mathrm{s}^{-1}$ and grey regions represent masked locations where the $\mathrm{S} / \mathrm{N}$ was not high enough to properly fit the emission lines. At most locations the uncertainties are smaller than $10 \mathrm{~km} \mathrm{~s}^{-1}$.

The emission-line ratio maps (Figs 5 and 6) show that the lowest ionization gas is located at the blob regions, while the high ionization gas is seen at the nucleus and and to the east. This result is consistent with the X-ray images of NGC 4395 presented by Akyuz et al. (2013), which show that the (2-12) keV emission peak at the nucleus, while lower energy $(0.2-2 \mathrm{keV})$ emission is seen mainly to the east of the nucleus.

The derived electron densities for NGC 4395 (Fig. 5) show values larger than $1300 \mathrm{~cm}^{-3}$ at the nucleus and the smallest values are observed at the blob region, which is also consistent with the observed ionization pattern. The $N_{\mathrm{e}}$ value derived for the nucleus of NGC 4395 is larger than the mean value observed for Seyfert nuclei using long-slit spectroscopy $\left(\sim 500 \mathrm{~cm}^{-3}-\right.$ e.g. Dors et al. 2014). This difference may be related to the fact that the values derived from long-slit spectra are based on larger apertures that include the circumnuclear region. Indeed, the $N_{\mathrm{e}}$ map for NGC 4395 is consistent with the range of values derived for other Seyfert galaxies using IFS (e.g. Freitas et al. 2018; Kakkad et al. 2018).
In a recent work, Kakkad et al. (2018) presented electron density maps for the inner few kiloparsec of 13 nearby active galaxies, derived from IFS and obtained as part of the Siding Spring Southern Seyfert Spectroscopic Snapshot Survey (S7). For all galaxies, they found highest values of $N_{\mathrm{e}}$ at the nucleus and smaller values at the circumnuclear region. The $N_{\mathrm{e}}$ map for NGC 4395 shows a similar behaviour.

\subsection{Diagnostic diagrams}

To investigate the gas excitation, line-ratio diagnostic diagrams are frequently used. The most popular one is a plot of [O III $] \lambda 5007 / \mathrm{H} \beta$ versus $[\mathrm{N}$ II] $\lambda 6584 \mathrm{H} \alpha$ line ratios, which is one of the Baldwin, Phillips \& Terlevich (BPT) diagrams (Baldwin, Phillips \& Terlevich 1981). Several alternative diagrams for distinct spectral bands and new calibrations to separate distinct classes have been proposed since the original work (e.g. Veilleux \& Osterbrock 1987; Allen, Dopita \& Tsvetanov 1998; Larkin et al. 1998; Kewley, Heisler 

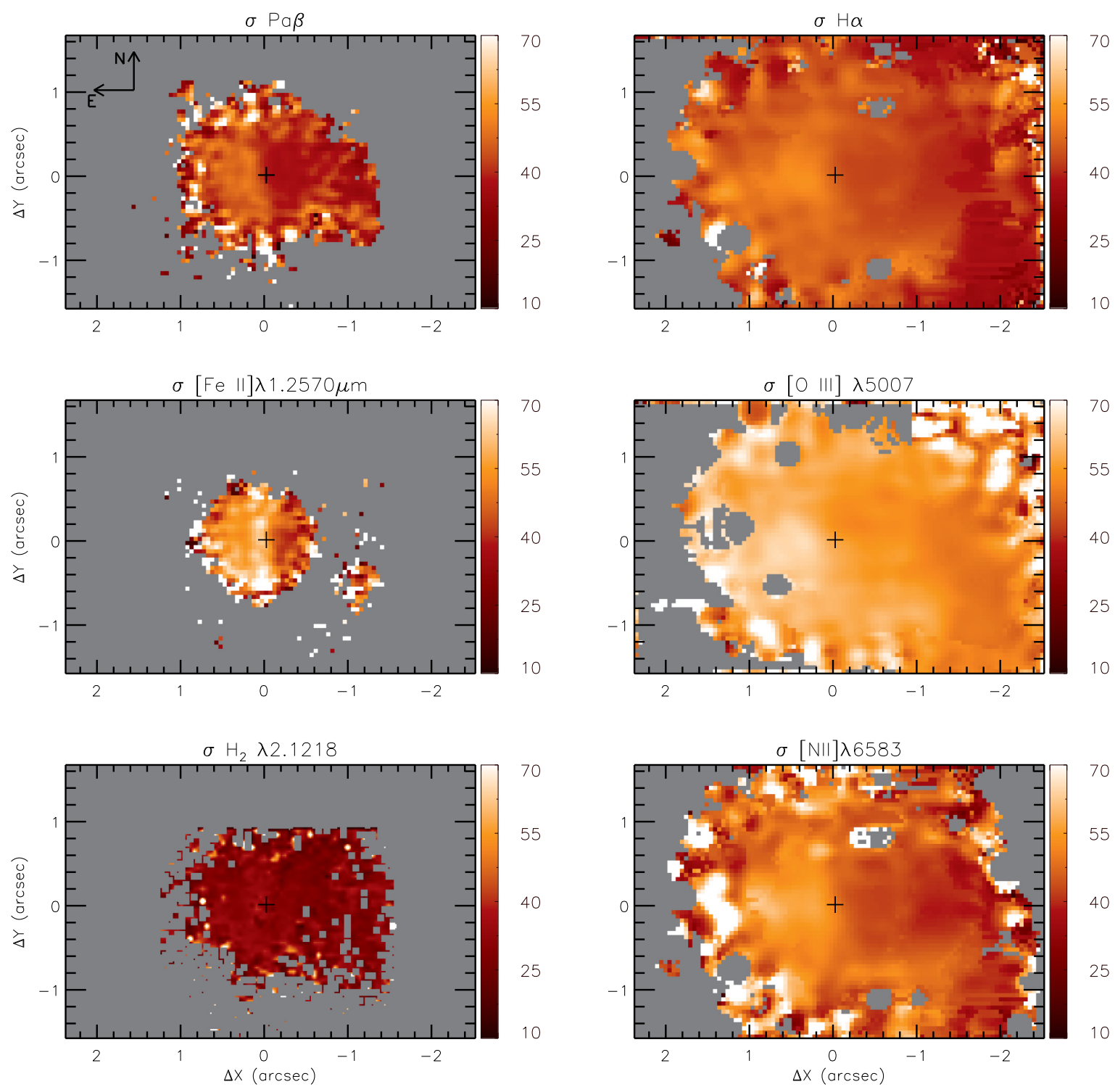

Figure 8. $\sigma$ maps for the same emission lines of Fig. 7. The colour bars show the $\sigma$ values in units of km s${ }^{-1}$.

\& Dopita 2001; Reunanen, Kotilainen \& Prieto 2002; Kauffmann et al. 2003; Rodríguez-Ardila et al. 2004; Kewley et al. 2006; Feltre, Charlot \& Gutkin 2016).

So far, most of the applications of diagnostic diagrams to the study of galaxies are based on single aperture spectra, and thus with very limited spatial information. Some recent studies have been using IFS to construct two-dimensional diagnostic diagrams (e.g. Sarzi et al. 2010; Colina et al. 2015; Sanchez et al. 2015; Belfiore et al. 2016), which reveal the presence of extra-nuclear kiloparsec scale low-ionization emission-line regions (LIERs) in both starforming and quiescent galaxies. Some studies have concluded that this LIER emission may be due to hot, evolved (post-asymptotic giant branch, post-AGB) stars and not due to the central ionizing source (Cid Fernandes et al. 2011; Singh et al. 2013; Stasińska et al. 2015; Belfiore et al. 2016).

In order to better map the gas excitation we have built the emission-line ratio diagnostic diagrams shown in Fig. 9. Each point corresponds to one spaxel. The blue lines, obtained from Kewley et al. (2006), are the dividing lines between H II-type galaxies (left) and AGN (right). The dotted line from Cid Fernandes et al. (2010) separates the region occupied by Seyferts (above the line) and LINERs and LIERs (below the line). The red and green diamonds shown in this figure are for the nucleus and blob region, respectively, as obtained from the measurements of the emission-line fluxes using the spectra shown in Fig. 2. As seen in Fig. 9, NGC 4395 shows intensity-line ratios over the whole GMOS FoV located in the AGN side of the $[\mathrm{O} \mathrm{III}] / \mathrm{H} \beta$ versus $[\mathrm{N} \mathrm{II}] / \mathrm{H} \alpha$ diagram. However, the region occupied by NGC 4395 is displaced to the left (smaller values of $[\mathrm{N} \mathrm{II}] / \mathrm{H} \alpha$ ), in comparison to classical Seyfert galaxies (e.g. Cid Fernandes et al. 2010; Freitas et al. 2018). This is an already known effect for AGN in dwarf galaxies, caused by the low gas metallicities observed in these objects (Kraemer et al. 1999; Cedrés \& Cepa 2002; Ludwing et al. 2012).

Further support for the results obtained using the optical line ratios can be gathered from IR diagnostic diagrams. In particular, the line flux ratios [Fe II] $\lambda 1.2570 \mu \mathrm{m} / \mathrm{Pa} \beta$ versus 

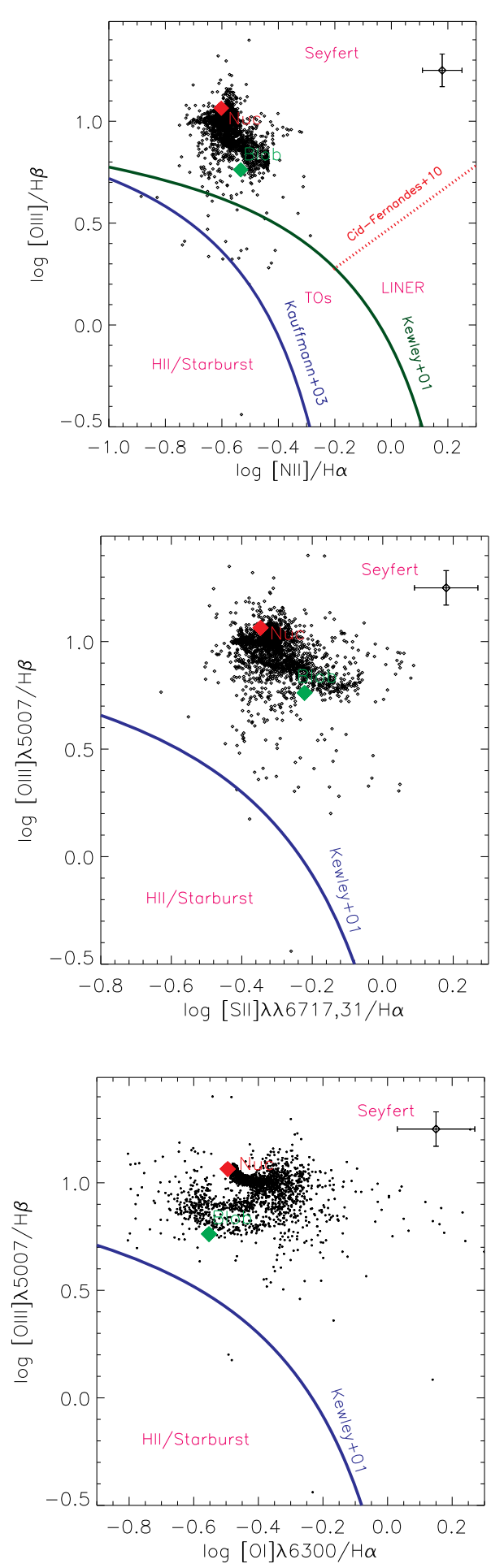

Figure 9. $[\mathrm{O} I I I] / \mathrm{H} \beta$ versus $[\mathrm{N} I I] / \mathrm{H} \alpha$ (top), $[\mathrm{O} \mathrm{III}] / \mathrm{H} \beta$ versus $[\mathrm{S} \mathrm{II}] / \mathrm{H} \alpha$ (middle), and $[\mathrm{O} \mathrm{III}] / \mathrm{H} \beta$ versus $[\mathrm{O} \mathrm{I}] / \mathrm{H} \alpha$ (bottom) diagnostic diagrams for NGC 4395 obtained from the GMOS data. The red and green diamonds are obtained by measuring the line ratios using the spectra shown in Fig. 2 for the nucleus and blob region, respectively. The uncertainties in the line flux ratios for the integrated spectra of the nucleus and blob are comparable to the size of the points and thus are not shown in the diagrams. The error bars represent the mean uncertainties in the line ratios.

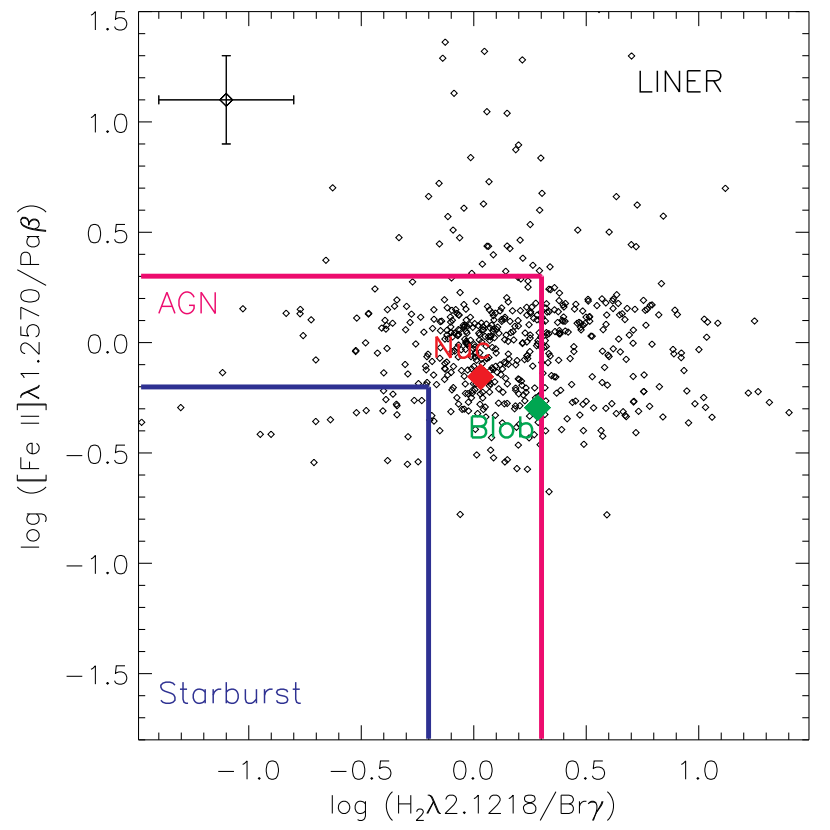

Figure 10. $[\mathrm{Fe} \mathrm{II}] \lambda 1.2570 \mu \mathrm{m} / \mathrm{Pa} \beta$ versus $\mathrm{H}_{2} \lambda 2.1218 \mu \mathrm{m} / \mathrm{Br} \gamma$ diagnostic diagram. The red and green diagram represent line ratios for the nucleus and the blob region, as obtained from the spectra shown in Fig. 2. The blue and magenta lines delimit regions with ratios typical of starbursts, Seyferts, and LINERs. The uncertainties in the line flux ratios for the integrated spectra of the nucleus and blob are similar to the size of the points and thus are not shown in the plot. The error bars represent the mean uncertainties in the line ratios.

$\mathrm{H}_{2} \lambda 2.1218 \mu \mathrm{m} / \mathrm{Br} \gamma$, shown in Fig. 10, also distinguish the excitation source among starburst activity, Seyfert activity, and LINERs (e.g. Larkin et al. 1998; Rodríguez-Ardila et al. 2004; RodríguezArdila et al. 2005; Riffel et al. 2010b). In this diagram, Starburst/H II galaxies occupy the bottom left corner of the plot, with both line ratios $<0.6$, LINERS show both ratios larger than 2 and Seyfert nuclei are in the region between the Starburst and LINER locations, with both line ratios in the range 0.6-2 (e.g. Rodríguez-Ardila et al. 2005; Riffel et al. 2013).

As for the optical diagram, the [Fe II] $\lambda 1.2570 \mu \mathrm{m} / \mathrm{Pa} \beta$ versus $\mathrm{H}_{2} \lambda 2.1218 \mu \mathrm{m} / \mathrm{Br} \gamma$ (Fig. 10) reveals that most locations of the central region of NGC 4395 have flux-line ratios typical of Seyfert galaxies. At some locations, the $\mathrm{H}_{2} / \mathrm{Br} \gamma$ line ratio falls in the LINER region of the diagram. It should be noticed that the lines delimiting the different regions in the $[\mathrm{Fe}$ II] $\lambda 1.2570 \mu \mathrm{m} / \mathrm{Pa} \beta$ versus $\mathrm{H}_{2} \lambda 2.1218 \mu \mathrm{m} / \mathrm{Br} \gamma$ are based on fluxes measured for the nuclear spectra of galaxies. Indeed, a recent work by Colina et al. (2015) using IFS revealed that the $\mathrm{H}_{2} \lambda 2.1218 \mu \mathrm{m} / \mathrm{Br} \gamma$ ratio can be higher for the extra-nuclear region of AGN-dominated sources, reaching values of up to 8 . Indeed both the nucleus and blob region present line ratios in the Seyfert region of the diagram, as obtained from the measurements of emission-line fluxes using the spectra shown in Fig. 2.

We conclude that the nuclear and circumnuclear region mapped by our observations are compatible with gas ionization by the central source.

\subsection{Molecular hydrogen and iron emission origin}

The $\mathrm{H}_{2}$ and [Fe II] emission lines can be excited by thermal and non-thermal processes. The former is due to gas heating by shocks 


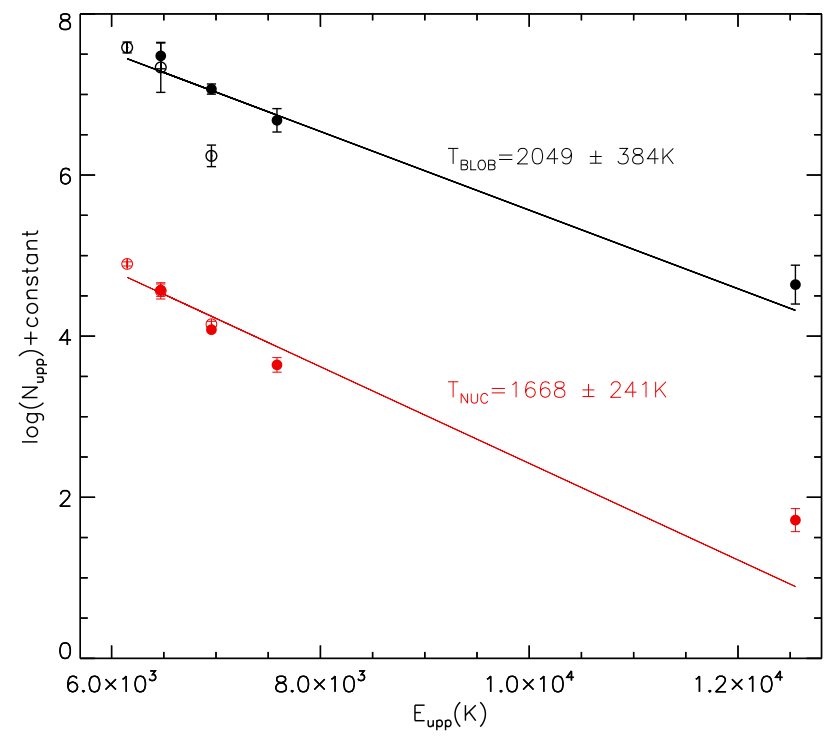

Figure 11. Relation between $N_{\text {upp }}=F_{\mathrm{i}} \lambda_{\mathrm{i}} / A_{\mathrm{i}} g_{\mathrm{i}}$ and $E_{\text {upp }}=T_{i}$ for the $\mathrm{H}_{2}$ emission lines for thermal excitation at the nucleus and the blob. Ortho $(S)$ transitions are shown as filled circles and para $(Q)$ transitions as filled circles.

or X-rays (e.g. from an AGN), the latter is by soft- or far-UV absorption, at dense clouds $\left(>10^{4} \mathrm{~cm}^{-3}\right)$ (Sternberg \& Dalgarno 1989). As discussed in Section 4.2, all line-ratio diagnostic diagrams for NGC 4395 suggest that the observed emission from all locations is due to gas excited by the central AGN.

We can further investigate the origin of $\mathrm{H}_{2}$ emission using the $\mathrm{H}_{2} \lambda 2.2477 / \mathrm{H}_{2} \lambda 2.1218$ line ratio (Mouri 1994; Reunanen et al. 2002; Storchi-Bergmann et al. 2009). For values ranging from 0.1 to 0.2 the $\mathrm{H}_{2}$ emission is due to thermal processes while values of $\approx 0.55$ is attributed to non-thermal processes. Using the spectra shown in Fig. 2 , we obtain $\mathrm{H}_{2} \lambda 2.2477 / \lambda 2.1218 \approx 0.13$ and $\approx 0.14$ for the nucleus and blob region, respectively. This supports an origin of the $\mathrm{H}_{2}$ line emission by thermal processes, possibly associated to the AGN. The $\mathrm{H}_{2}$ excitation temperature $T_{\text {exc }}$ can be obtained using the measured fluxes of the $\mathrm{H}_{2}$ emission lines together with the following expression (Scoville et al. 1982):

$\log \left(\frac{F_{\mathrm{i}} \lambda_{\mathrm{i}}}{A_{\mathrm{i}} g_{\mathrm{i}}}\right)=$ constant $-\frac{T_{\mathrm{i}}}{T_{\mathrm{exc}}}$,

where $F_{\mathrm{i}}$ is the flux of the $i$ th $\mathrm{H}_{2}$ line, $\lambda_{i}$ is its wavelength, $A_{i}$ is the spontaneous emission coefficient, $g_{i}$ is the statistical weight of the upper level of the transition, $T_{i}$ is the energy of the level expressed as a temperature, and $T_{\mathrm{exc}}$ is the excitation temperature. The equation (2) assumes a ratio of transitions ortho:para of 3:1 and is valid only for thermal equilibrium.

Fig. 11 shows a plot of $\mathrm{N}_{\text {upp }}=F_{i} \lambda_{i} / A_{i} g_{i}$ (plus an arbitrary constant) versus $E_{\text {upp }}=T_{i}$, where filled symbols represent ortho transition and open symbols para transitions. The best linear fit is shown as a continuous line, for the nucleus (shown in red) and the blob region (shown in black). The resulting excitation temperatures are $T_{\mathrm{NUC}}=1923 \pm 294 \mathrm{~K}$ and $T_{\mathrm{BLOB}}=$ $2002 \pm 265 \mathrm{~K}$ for the nucleus and blob, respectively. This suggests that the $\mathrm{H}_{2}$ emitting gas is in thermal equilibrium, supporting that $\mathrm{H}_{2}$ excitation mechanism is due to thermal excitation by the AGN the main responsible by molecular hydrogen emission.
The $[\mathrm{Fe} I \mathrm{I}] \lambda 1.25 \mu \mathrm{m} /[\mathrm{P} \mathrm{II}] \lambda 1.188 \mu \mathrm{m}$ line ratio is an important tracer of the origin of the [Fe II] emission (Oliva et al. 2001). Both [Fe II] and [P II] have emission lines close in wavelengths and similar excitation temperatures. Moreover, their parent ions have similar ionization potentials and radiative recombination coefficients. H II regions present $[\mathrm{Fe} I \mathrm{II}] \lambda 1.25 \mu \mathrm{m} /[\mathrm{P} \mathrm{II}] \lambda 1.188 \mu \mathrm{m} \approx 2$, while for supernovae remnants where the $[\mathrm{Fe} \mathrm{II}]$ emission is due to shocks, the above line ratio reaches values larger than 20 (Oliva et al. 2001). Thus, values larger than 2 for the line ratio of [Fe II]/[P II] indicate that shocks have passed through the gas destroying the dust grains, releasing the iron in the environment. For values higher than 20, shocks are the dominant excitation mechanism (Oliva et al. 2001; Storchi-Bergmann et al. 2009). In NGC 4395 (see Fig. 6), at most locations the $[\mathrm{Fe} \mathrm{II}] \lambda 1.25 \mu \mathrm{m} /[\mathrm{P} \mathrm{II}] \lambda 1.188 \mu \mathrm{m}$ ratio values range from 2 to 7 , with some high values (of up to 12) observed at distances larger than 0.5 from the nucleus, where the uncertainty in the line ratio is high. From the flux values quoted in Table 1, we obtain [Fe II]/[P II] of $\approx 6.5$ for the nucleus and $\approx 3.5$ for the blob region. These values indicate that the $[\mathrm{Fe}$ II] emission in NGC 4395 is mainly due gas photoionized by its AGN radiation, but some contribution by shocks cannot be discarded.

\subsection{The broad-line emission}

The FWHM of the broad component measured for the $\mathrm{H}$ recombination lines using the nuclear spectrum shown in Fig. 2 is $785 \pm 40 \mathrm{~km} \mathrm{~s}^{-1}$. This value is smaller than that derived by Kraemer et al. (1999), who obtained FWHM $\sim 1500 \mathrm{~km} \mathrm{~s}^{-1}$ for the $\mathrm{H} \beta$ broad component using moderate resolution (FWHM 5-8 $\mathrm{A}$ ) spectroscopy with a $3 \mathrm{~m}$ telescope at the Lick Observatory. Using the spectrum of NGC 4395 obtained by the SDSS (Abolfathi et al. 2018) we derive FWHM $=910 \mathrm{~km} \mathrm{~s}^{-1}$ for $\mathrm{H} \alpha$ broad component, which is more similar to the value obtained from GMOS spectra. The origin of the discrepancies among the values derived for the FWHM using different observations is not clear. A possible explanation is that the discrepancies could be due to the variability of the BLR emission, making it difficult to properly constrain the width of the broad-line component when its flux is low. In addition, we notice that the centroid of the broad-line components is blueshifted by $45 \mathrm{~km} \mathrm{~s}^{-1}$ relative to the velocities for the narrow lines, suggesting outflows in the BLR.

The observed luminosity of the broad $\mathrm{H} \alpha$ component can be used to estimate the AGN bolometric luminosity $\left(L_{\mathrm{bol}}\right)$ as (Greene \& Ho 2005b, 2007)

$L_{\text {bol }}=2.34 \times 10^{44}\left(L_{\mathrm{H} \alpha} / 10^{42}\right)^{0.86}$.

Using $L_{\mathrm{H} \alpha}=(1.2 \pm 0.2) \times 10^{38} \mathrm{erg} \mathrm{s}^{-1}$, as measured from the nuclear spectrum shown in Fig. 2, we obtain $L_{\text {bol }}=(9.9 \pm 1.4) \times$ $10^{40} \mathrm{erg} \mathrm{s}^{-1}$. This is larger than that derived by integrating the global spectral energy distribution of the nucleus of NGC 4395 $\left(L_{\text {bol }}=1.9 \times 10^{40} \mathrm{erg} \mathrm{s}^{-1} ;\right.$ Moran et al. 1999). The AGN bolometric luminosity can also be be estimated from the the hard X-ray luminosity $\left(L_{X}\right)$ by Ichikawa et al. (2017)

$\log L_{\text {bol }}=0.0378\left(\log L_{\mathrm{X}}\right)^{2}-2.03 \log L_{\mathrm{X}}+61.6$.

For NGC 4395, the hard X-ray (14-195 keV) luminosity is $L_{\mathrm{X}} \approx$ $(6.3 \pm 0.6) \times 10^{40} \mathrm{erg} \mathrm{s}^{-1}$ as detected in the Swift/BAT survey (Kyuseok et al. 2018). Thus, the estimated bolometric luminosity of the AGN of NGC 4395 is $L_{\text {bol }}=(4.9 \pm 0.4) \times 10^{41} \mathrm{erg} \mathrm{s}^{-1}$. The value for the bolometric luminosity derived from the $\mathrm{H} \alpha$ broad component is then intermediate. 

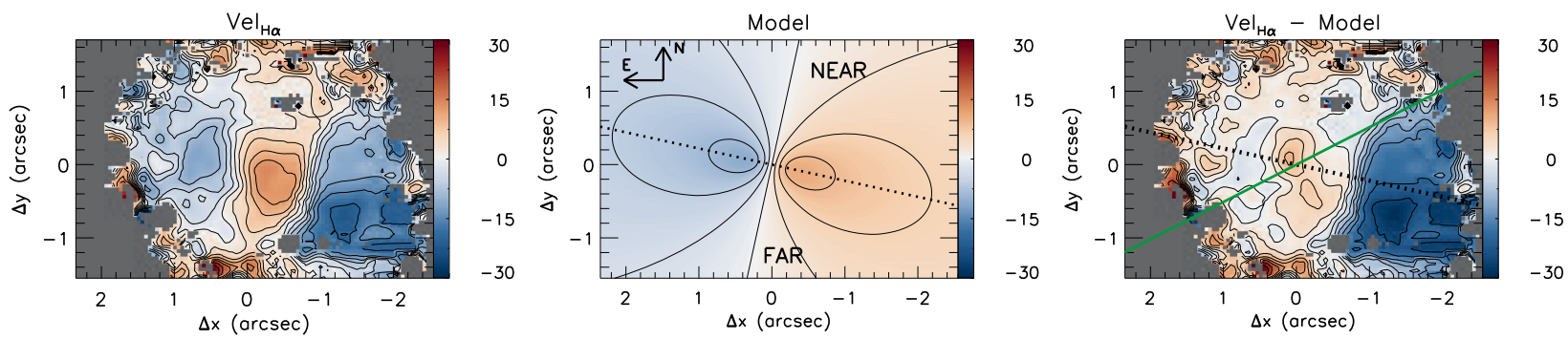

Figure 12. From left to right: $\mathrm{H} \alpha$ velocity field, rotating disc model for the $\mathrm{H} \alpha$ velocity field, and residual map (between the observed, modelled velocities). The colour bars show the velocities in $\mathrm{km} \mathrm{s}^{-1}$. The dotted line displays the orientation of the line of nodes and the continuous green line shows the orientation of the photometric major axis of the galaxy from Jarrett et al. (2003). The near and far side of the galaxy are indicated in the middle panel.

We can estimate the mass of the central SMBH by (Greene \& Ho 2007)

$$
\begin{aligned}
\left(\frac{M_{\mathrm{BH}}}{\mathrm{M}_{\odot}}\right)= & \left(3.0_{-0.5}^{+0.6}\right) \times 10^{6}\left(\frac{L_{\mathrm{H} \alpha}}{10^{42} \mathrm{ergs}^{-1}}\right)^{0.45 \pm 0.03} \\
& \times\left(\frac{\mathrm{FWHM}_{\mathrm{H} \alpha}}{10^{3} \mathrm{~km} \mathrm{~s}^{-1}}\right)^{2.06 \pm 0.06} .
\end{aligned}
$$

Using the $\mathrm{H} \alpha$ luminosity and FWHM for the broad component, listed above, we obtain $M_{\mathrm{BH}}=\left(2.5_{-0.8}^{+1.0}\right) \times 10^{5} \mathrm{M}_{\odot}$, which is consistent with the values previously estimated using reverberation mapping $\left((3.6 \pm 1.1) \times 10^{5} \mathrm{M}_{\odot}\right.$; Peterson et al. 2005) and by modelling the molecular gas dynamics $\left(4_{-3}^{+8} \times 10^{5} \mathrm{M}_{\odot}\right.$; Den Brok et al. 2015).

\subsection{Gas kinematics}

The velocity fields shown in Fig. 7 show redshifts to the west of the nucleus and the blueshifts to the east. This behaviour suggests the presence of a disc rotation component with the line of nodes oriented approximately along the east-west direction. Another possible interpretation for the origin of this kinematic component could be the presence of a biconical outflow driven by the central AGN of NGC 4395. However, the velocity dispersion maps (Fig. 8) show small values $\left(\lesssim 50 \mathrm{~km} \mathrm{~s}^{-1}\right)$ in all locations and an enhancement of the $\sigma$ values is expected within the cone in case of the presence of outflows due to the interaction of the winds launched from the AGN and the ambient gas (e.g. Riffel et al. 2006; Freitas et al. 2018). Because of that we conclude that the gas kinematics close to the nucleus is due to rotation of gas in the plane of the disc. In addition, non-circular motions of gas are seen to the west, as indicated by the blueshifts observed at the blob region.

We fitted a simple analytical model to the $\mathrm{H} \alpha$ centroid velocity field of NGC 4395, assuming that the gas has circular orbits in a plane of the galaxy, as done in previous works (e.g. Couto et al. 2013; Schnorr-Müller et al. 2014a, b; Lena et al. 2015, 2016; Brum et al. 2017; Hekatelyne et al. 2017). The expression for the line-ofsight velocity is given by Bertola, Bettoni \& Danziger (1991):

$$
\begin{aligned}
& V_{\text {mod }}(R, \Psi)=V_{\mathrm{s}}+ \\
& \frac{A R \cos \left(\Psi-\Psi_{0}\right) \sin (i) \cos ^{p}(i)}{\left\{R^{2}\left[\sin ^{2}\left(\Psi-\Psi_{0}\right)+\cos ^{2}(i) \cos ^{2}\left(\Psi-\Psi_{0}\right)\right]+C_{o}^{2} \cos ^{2}(i)\right\}^{p / 2}},
\end{aligned}
$$

where $V_{\mathrm{s}}$ is the systemic velocity of the galaxy, $A$ is the velocity amplitude, $\Psi_{0}$ is the position angle of the line of nodes, $i$ is the disc inclination in relation to the plane of the sky, $C_{0}$ is a concentration parameter, defined as the radius where the rotation curve reaches 70 per cent of its velocity amplitude, the parameter $p$ measures the slope of the rotation curve where it flattens, in the outer region of the galaxy and it is limited in the range $1 \leq p \leq 3 / 2$ (for $p=1$ the rotation curve at large radii is asymptotically flat while for $p=$ $3 / 2$ the system has a finite mass), and $R$ is the radial distance to the nucleus projected in the plane of the sky with the corresponding position angle $\Psi$.

We used an interactive data language (IDL) routine to fit the above equation to the observed $\mathrm{H} \alpha$ velocity field using the MPFITFUN routine (Markwardt et al. 2009) to perform the non-linear leastsquares fit. As all lines show similar velocity fields, we chose the $\mathrm{H} \alpha$ to perform the fit, as it is the strongest emission line observed at most locations of the galaxy. During the fit, the position of the kinematical centre was kept fixed to the location of the peak of the continuum emission, we fixed $p=1.5$, as done in previous works (e.g. Brum et al. 2017; Hekatelyne et al. 2017) and the inclination of the disc was fixed to $i=37^{\circ}$, as derived by Den Brok et al. (2015) constructing dynamical models for the gas velocity field measured using NIFS data. As the blueshifts observed at the blob region trace non-circular motions, we excluded this region during the fit, by excluding all spaxels west of the nucleus observed in blueshifts.

Fig. 12 shows the observed $\mathrm{H} \alpha$ velocity field in the left-hand panel, the best-fitting model in the middle panel and the residual map, obtained by the difference between the observed velocities and the model, is shown in the right-hand panel. Although the velocity amplitude in the central region of NGC 4395 is small, the rotation disc model is a very good representation of the observed gas velocity field, with the exception of the blob region. The residuals map shows values very close to zero $\left(<5 \mathrm{~km} \mathrm{~s}^{-1}\right)$ at most other locations.

The parameters derived from the fit are: $V_{s}=326 \pm 7 \mathrm{~km} \mathrm{~s}^{-1}$ (corrected for the heliocentric rest frame), $A=16 \pm 3 \mathrm{~km} \mathrm{~s}^{-1}, C_{0}$ $=0^{\prime \prime} .21 \pm 0^{\prime} .03(4.2 \pm 0.6 \mathrm{pc}), \Psi_{0}=78^{\circ} \pm 3^{\circ}$. The systemic velocity resulting from the modelling is consistent with the value derived from H I $21 \mathrm{~cm}$ line emission observations of NGC 4395 ( $319 \pm 1 \mathrm{~km} \mathrm{~s}^{-1}$, Haynes et al. 1998), while the orientation of the line of nodes is displaced by $39^{\circ}$ in relation to the value obtained for the large-scale disc using $K$ band images from the 2MASS Extended Source Catalog (Jarrett et al. 2003). A possible interpretation for the difference between the kinematic position angle in the inner region and the orientation of the large-scale disc is that it is due to a kinematically distinct core (KDC), as observed for other galaxies (Emsellem, Krajnović \& Sarzi 2004; Raimundo et al. 2013, 2017; Davies et al. 2014). However, this interpretation should be taken with caution, as $\Psi_{0}$ value may not be properly constrained in our modelling due to the small FOV of the observations. 
We note that the concentration parameter $C_{0}$ is very small, only $4.2 \mathrm{pc}$, meaning that a very compact nuclear mass distribution is driving the motions of the gas in the central region. Indeed, the observed gas velocity field shows the maximum velocity amplitude very close to the nucleus at a distance $R \sim 00^{\prime \prime} 5(10 \mathrm{pc})$ from it. Assuming a simple Keplerian rotation, the mass contained within this radius can be obtained by $M=\frac{2 R V_{\mathrm{m}}^{2}}{G \sin ^{2} i}$, where $V_{\mathrm{m}}$ is the maximum projected velocity amplitude and $G$ is the Newton's gravitational constant. Using $V_{\mathrm{m}}=25 \mathrm{~km} \mathrm{~s}^{-1}$ and $i=37^{\circ}$, we obtain $M \approx 7.7 \times 10^{5} \mathrm{M}_{\odot}$. It is well known that NGC 4395 harbours a young (100-300 Myr) nuclear stellar cluster (Carson et al. 2015) and thus the derived above mass may be dominated by this stellar cluster (Filippenko, Ho \& Sargent 1993). The mass value derived above is somewhat smaller than that found by Filippenko \& Ho (2003) for the mass of the compact nucleus of NGC 4395 (within $3.9 \mathrm{pc} ; \sim 6.2 \times 10^{6} \quad \mathrm{M}_{\odot}$ ) and is about twice the mass of the SMBH as derived by dynamical models (Den Brok et al. 2015) and reverberation mapping (Peterson et al. 2005).

\subsection{The nature of the 'blob'}

In the residual map, at the blob region an excess blueshift of $\approx 30 \mathrm{~km} \mathrm{~s}^{-1}$ is observed. Considering that the spiral arms seen in the large-scale image (Fig. 1) are of the trailing type, and using the observed velocity fields (Fig. 7) we conclude that the south-southwest is the far side of the galaxy and the north-north-east is the near side. The blob presents a small velocity dispersion, on average, of $40 \mathrm{~km} \mathrm{~s}^{-1}$ (Fig. 8) and lower ionization gas, as indicated by the line ratio maps of Fig. 5, indicating that the gas is located at the plane of the galaxy.

Two alternatives can be suggested to explain the origin of this structure. In the first case, it is a result of a minor merger of a gas-rich small satellite. Similar scenarios are found for other galaxies. For example, Fischer et al. (2015) report an ongoing minor merger with a gas-rich dwarf galaxy inflowing towards the nucleus of Mrk 509 and Mezcua et al. (2018) found that a minor merger identified in the Seyfert galaxy NGC 5252 shows radio emission consistent with an intermediate-mass black hole with mass $10^{3.5}<M_{\mathrm{BH}} \lesssim 2 \times 10^{6}$ $\mathrm{M}_{\odot}$. The detection of a minor merger in a dwarf AGN opens new window to understand the feeding process of small black holes.

Another possible scenario for the origin of the blob is that it could be a low-mass/low-metallicity cosmic gas cloud accreted on to NGC 4395, creating a localized starburst with distinct kinematics and lowered gas-phase metallicities (Sánchez Almeida et al. 2015). This scenario is expected in the Lambda cold dark matter context (Ceverino et al. 2016).

\subsection{Estimating the mass inflow rate}

We can estimate the mass of the ionized gas in the blob region as

$M_{\mathrm{H} \text { II }}=m_{\mathrm{p}} n_{\mathrm{e}} V f$,

where $m_{\mathrm{p}}$ is the proton mass, $n_{\mathrm{e}}$ is the electron density, $V$ is the volume of the emitting region, and $f$ is the filling factor. The filling factor can be estimated by

$L_{\mathrm{H} \alpha} \sim f N_{\mathrm{e}}^{2} j_{\mathrm{H} \alpha}(T) V$,

where $j_{\mathrm{H} \alpha}=3.534 \times 10^{-25} \mathrm{~cm}^{-3} \mathrm{~s}^{-1}$ for an electron temperature of $T=10000 \mathrm{~K}$ (Osterbrock \& Ferland 2006) and $L_{\mathrm{H} \alpha}$ is the $\mathrm{H} \alpha$ luminosity emitted by a volume $V$. By replacing equation (8) into equation (7), we obtain

$M_{\mathrm{H} \text { II }}=\frac{m_{\mathrm{p}} L_{\mathrm{H} \alpha}}{N_{\mathrm{e}} j_{\mathrm{H} \alpha}(T)}$.

As the spatial resolution of our near-IR data is better than that of the GMOS data and the dust extinction in the near-IR is less important, we use the theoretical ratio between the $\mathrm{H} \alpha$ and $\mathrm{Br} \gamma\left(L_{\mathrm{Br} \gamma}\right)$ luminosities, as estimated adopting the case B of recombination for $T=10000 \mathrm{~K}$ at the low-density limit $\left(L_{\mathrm{H} \alpha}=102.1 L_{\mathrm{Br} \gamma}\right.$; Osterbrock \& Ferland 2006). By replacing $L_{\mathrm{H} \alpha}, m_{\mathrm{p}}$, and $j_{\mathrm{H} \alpha}(T)$ in equation (9) and rewriting it, we obtain:

$$
\left(\frac{M_{\mathrm{H} \text { II }}}{\mathrm{M}_{\odot}}\right)=2.9 \times 10^{19}\left(\frac{F_{\mathrm{Br} \gamma}}{\operatorname{erg~s}^{-1} \mathrm{~cm}^{-2}}\right)\left(\frac{D}{\mathrm{Mpc}}\right)^{2}\left(\frac{\mathrm{cm}^{-3}}{N_{\mathrm{e}}}\right),
$$

where $F_{\mathrm{Br} \gamma}$ is the flux of the $B r \gamma$ emission line.

The total $\mathrm{Br} \gamma$ flux emitted by the blob region is $F_{\mathrm{Br} \gamma} \approx$ $1.3 \times 10^{-16} \mathrm{erg} \mathrm{cm}^{-2} \mathrm{~s}^{-1}$, measured within a circular aperture of 0. 5 radius centred at the blob. As the reddening in the blob regions is very small, the correction of the above flux by extinction is negligible. The average electron density in the blob region is $480 \mathrm{~cm}^{-3}$ as determined from the [S II] 6717,31 line ratio (see Fig. 5 and Section 3.3.1). The mass of the ionized gas is therefore $M_{\mathrm{H} \text { II }} \approx 150 \mathrm{M}_{\odot}$.

As discussed above, we interpret the blueshifts seen at the blob region (24 pc west of the nucleus) as resulting from emission of gas inflowing towards the nucleus. The observed blueshifts are $\sim 30 \mathrm{~km} \mathrm{~s}^{-1}$ (Fig. 12). Assuming that the inflows occur in the plane of the galaxy, the deprojected inflowing velocity is $v_{\text {in }} \approx 49 \mathrm{~km} \mathrm{~s}^{-1}$, using the disc inclination of $i=37^{\circ}$. If the blob is moving directly to the centre with this velocity it would take $t_{\mathrm{d}} \approx 4.8 \times 10^{5} \mathrm{yr}$ to reach the nucleus, assuming a distance of the nucleus of $1^{\prime \prime} .2$ for the blob. The corresponding mass inflow rate of ionized gas is $\phi=\frac{M_{\mathrm{H}}}{t_{\mathrm{d}}} \approx 3.2 \times 10^{-4} \mathrm{M}_{\odot} \mathrm{yr}^{-1}$. This value is one order of magnitude smaller than those found for nearby Seyfert galaxies $\left(10^{-3}-10^{-1} \mathrm{M}_{\odot} \mathrm{yr}^{-1}\right.$; e.g. Riffel 2013). Considering that NGC 4395 is a dwarf low-mass galaxy, a smaller value for the inflow rate is expected.

\subsection{Molecular and ionized gas surface mass density}

The fluxes of the $\mathrm{H}_{2} \lambda 2.12 \mu \mathrm{m}$ and $\mathrm{Br} \gamma$ emission lines can be used to estimate the mass of hot molecular and ionized gas, respectively. The mass of ionized gas was estimated using equation (10). The mass of hot molecular gas $\left(M_{\mathrm{H} 2}\right)$ can be estimated from (e.g. Scoville et al. 1982; Riffel et al. 2014, 2018):

$$
\left(\frac{M_{\mathrm{H} 2}}{M_{\odot}}\right)=5.0776 \times 10^{13}\left(\frac{F_{\mathrm{H} 2 \lambda 2.1218}}{\mathrm{erg} \mathrm{s}^{-1} \mathrm{~cm}^{-2}}\right)\left(\frac{D}{\mathrm{Mpc}}\right)^{2},
$$

where $F_{\mathrm{H} 2 \lambda 2.1218}$ is the flux for the $\mathrm{H}_{2} \lambda 2.1218 \mu \mathrm{m}$ emission line and a vibrational temperature of $T=2000 \mathrm{~K}$ was adopted (e.g. Riffel et al. 2008, 2018; Storchi-Bergmann et al. 2009; Riffel, StorchiBergmann \& Nagar 2010a; Schönel et al. 2014, 2017).

Considering the observed $E(B-V)$ values, the correction of the fluxes of the $K$-band lines due to dust extinction is small ( $<7$ per cent at all locations) and thus we use the observed fluxes to derive the gas masses. Calculating the molecular and ionized gas masses for each spaxel and integrating over the whole NIFS FoV, we obtain $M_{\mathrm{H} \mathrm{II}} \approx$ $5.9 \times 10^{4} \mathrm{M}_{\odot}$ and $M_{\mathrm{H} 2} \approx 2.2 \mathrm{M}_{\odot}$. To calculate $M_{\mathrm{H} \text { II }}$ we used the $N_{\mathrm{e}}$ values derived from the [S II] lines at each spaxel. The $M_{\mathrm{H} \text { II }}$ and $M_{\mathrm{H} 2}$ derived for NGC 4395 are about one order of magnitude smaller than the values derived for the inner few hundred of parsecs 

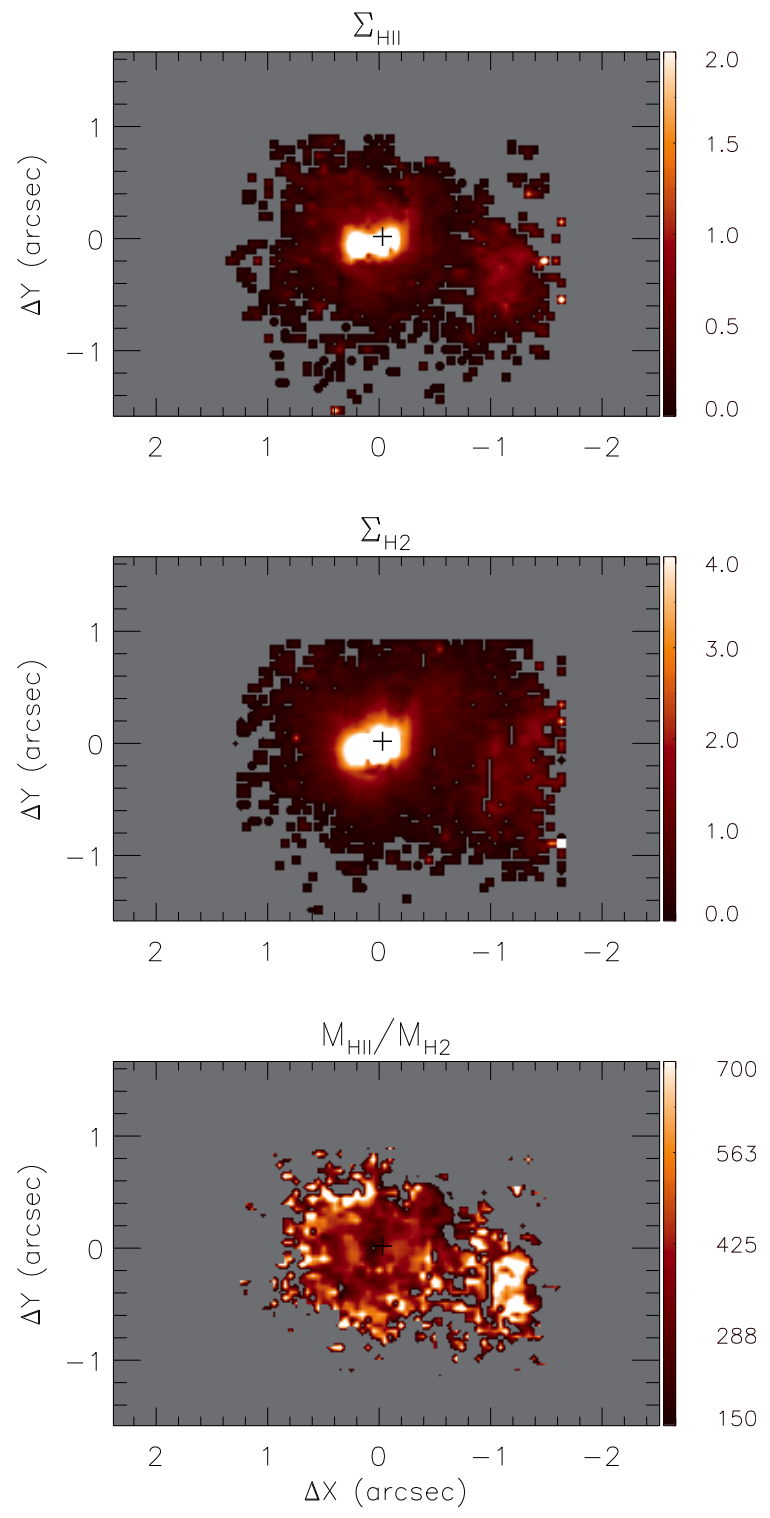

Figure 13. Surface mass density of ionized (top) and molecular (middle) gas and ratio between ionized and molecular gas masses (bottom). The colour bars show the surface mass densities in units of $\mathrm{M}_{\odot} \mathrm{pc}^{2}$ for the ionized gas and of $10^{-3} \mathrm{M}_{\odot} \mathrm{pc}^{2}$ for the molecular gas.

of nearby Seyfert galaxies $-3.5 \times 10^{4}<M_{\mathrm{H} \text { II }}<4.4 \times 10^{6} \mathrm{M}_{\odot}$ and $50<M_{\mathrm{H} 2}<2800 \mathrm{M}_{\odot}-$ using the same methodology (Riffel et al. 2018). However it should be noticed that the spatial coverage of the NIFS data on NGC 4395 (tens of parsecs) is smaller than those on the Seyfert galaxies of the above study.

A better way to trace the molecular and ionized gas content is by calculating their surface mass densities, which are less sensitive to the size of the FoV. We calculated the ionized and molecular gas masses spaxel-by-spaxel and constructed the surface mass density $(\Sigma)$ maps shown in Fig. 13. The ionized gas surface mass density $\left(\Sigma_{\mathrm{H} \text { II }}\right)$ show the highest values of $\sim 2 \mathrm{M}_{\odot} \mathrm{pc}^{-2}$ at the nucleus, while a secondary peak is seen at the blob region with $\Sigma_{\mathrm{H} \text { II }} \sim 1 \mathrm{M}_{\odot} \mathrm{pc}^{-2}$. The hot molecular gas mass surface density $\left(\Sigma_{\mathrm{H} 2}\right)$ shows a similar distribution, with values of $\sim 4 \times 10^{-3} \mathrm{M}_{\odot} \mathrm{pc}^{-2}$ and $\sim 1 \times 10^{-3}$ $\mathrm{M}_{\odot} \mathrm{pc}^{-2}$ for the nucleus and blob region, respectively. The values of $\Sigma_{\mathrm{H} \text { II }}$ and $\Sigma_{\mathrm{H} 2}$ observed for NGC 4395 are in the range of values typically observed for Seyfert galaxies (Riffel et al. 2018), for which $0.2<\Sigma_{\mathrm{H} \text { II }}<36 \mathrm{M}_{\odot} \mathrm{pc}^{-2} 0.2 \times 10^{-3}<\Sigma_{\mathrm{H} 2}<14 \times 10^{-3} \mathrm{M}_{\odot} \mathrm{pc}^{-2}$.

The bottom panel of Fig. 13 shows the ratio between ionized and molecular gas masses. The smallest values of $\sim 300$ are observed at the nucleus, while the highest values of up to 700 are seen at the blob region. The smaller ratio at the nucleus indicates that part of the molecular hydrogen is being dissociated by the radiation of the central AGN. Riffel et al. (2018) derived ratios ranging from $\sim 200$ to $\sim 8000$ for nearby Seyfert galaxies and thus, the values observed for NGC 4395 are consistent with their measurements, being similar to the lowest observed ratios.

\subsection{Feeding the AGN of NGC 4395}

The mass accretion rate to the AGN can be estimated to compare to the accretion rate necessary to power the AGN at the nucleus of NGC 4395, which can be derived by

$\dot{m}=\frac{L_{\text {bol }}}{c^{2} \eta}$,

where $L_{\mathrm{bol}}$ is the nuclear bolometric luminosity, $\eta$ is the efficiency of conversion of the rest mass energy of the accreted material into radiation, and $c$ is the light speed. $L_{\text {bol }} \approx 9.9 \times 10^{40} \mathrm{erg} \mathrm{s}^{-1}$, as estimated in Section 4.4. Assuming $\eta \approx 0.1$, which is a typical value for a 'standard' geometrically thin, optically thick accretion disc (e.g. Frank, King \& Raine 2002), we obtain a mass accretion rate of $\dot{m} \approx 2.5 \times 10^{-5} \mathrm{M}_{\odot} \mathrm{yr}^{-1}$. This value is about one order of magnitude smaller than the derived mass inflow rate, indicating that most of the gas that reach the central region of NGC 4395 is not used to power its AGN, but most probably is consumed by star formation. This conclusion is further supported by the detection of an young nuclear star cluster in NGC 4395 (Filippenko \& Ho 2003; Carson et al. 2015).

Considering the estimated masses of ionized and hot molecular gas $\left(M_{\mathrm{H} \text { II }} \approx 5.9 \times 10^{4} \mathrm{M}_{\odot}\right.$ and $\left.M_{\mathrm{H} 2} \approx 2.2 \mathrm{M}_{\odot}\right)$ and the derived mass accretion rate, we conclude that the ionized gas reservoir alone would be enough to feed the AGN of NGC 4395 for an activity cycle of $10^{7}-10^{8} \mathrm{yr}$. In addition, it is known that the ratio between the cold and hot molecular gas masses in the central regions of galaxies is in the range $10^{5}-10^{8}$ (Dale et al. 2005; Müller-Sánchez et al. 2006; Mazzalay et al. 2013) thus the total amount of gas in the inner $50 \mathrm{pc}$ of NGC 4395 may be at least of the order of $10^{5} \mathrm{M}_{\odot}$. Thus, the available mass reservoir, besides feed the AGN, may also be used to form new stars. This scenario is supported by the detection of a nuclear star cluster in NGC 4993 (Filippenko \& Ho 2003; Carson et al. 2015), as well as by studies of nearby Seyfert galaxies that reveal the evidence of recent star formation (e.g. Riffel et al. 2015a, 2017).

\section{CONCLUSIONS}

NGC 4395 is an interesting galaxy mostly for two reasons: (i) it is very nearby so we can get a very detailed look at a Seyfert galaxy, and (ii) it is a rare, confirmed dwarf AGN that could be a local analogue of small black holes that are growing in the early universe. We presented optical and near-IR IFS of the inner $65 \times 100 \mathrm{pc}^{2}$ of NGC 4395 at angular resolutions of $0{ }^{\prime} 2(4 \mathrm{pc})$ in the $J$-band, $00^{\prime \prime} 3$ (6pc) in the $K$ band, and 0.5 (10 pc) in the optical. This enabled us to estimate an extensive set of ionized and molecular gas properties, which are summarized and interpreted as follows: 
(i) The optical and near-IR emission-line flux distributions show extended emission. The line emission peaks at the nucleus but is also extended in a blob at 1".2 (24 pc) west of the nucleus. The line ratios at all locations suggest that the gas is ionized by high-energy photons from the AGN (as opposed to shocks);

(ii) The gas velocity fields are consistent with two kinematic components, one due to gas rotation at the plane of the disc of the galaxy plus another due to inflows of gas at the blob region;

(iii) The rotation kinematic component is well modelled by a simple analytical model, which suggests a very compact mass distribution, with the rotation curve reaching its maximum at only $10 \mathrm{pc}$ from the nucleus. We estimate a mass of $7.7 \times 10^{5} \mathrm{M}_{\odot}$ inside this radius, which is about twice the estimated mass of the $\mathrm{SMBH}$ of NGC 4395 and similar to the mass of the nuclear star cluster previously known;

(iv) Gas in the blob region is blueshifted by $\approx 30 \mathrm{~km} \mathrm{~s}^{-1}$ compared to the surrounding material. This is interpreted as gas flowing towards the nucleus at a rate of $\approx 3.2 \times 10^{-4} \mathrm{M}_{\odot} \mathrm{yr}^{-1}$. The origin of the inflowing material is not clear, but it may be an ongoing minor merger of a gas-rich small galaxy, or the accretion of a lowmetallicity cosmic cloud.

(v) The mean surface mass density for the ionized and molecular gas are in the ranges (1-2) $\mathrm{M}_{\odot} \mathrm{pc}^{-2}$ and (1-4) $\times 10^{-3} \mathrm{M}_{\odot} \mathrm{pc}^{-2}$. The ratio between the ionized and molecular gas are $\sim 300$ at the nucleus and $\sim 700$ at the blob region. The gas surface densities derived for NGC 4395 lie at the lower end of the range of values observed for typical Seyfert galaxies.

(vi) The mass of ionized and hot molecular gas within the inner $50 \mathrm{pc}$ are $M_{\mathrm{H} \text { II }} \approx 5.9 \times 10^{4} \mathrm{M}_{\odot}$ and $M_{\mathrm{H} 2} \approx 2.2 \mathrm{M}_{\odot}$, respectively. Considering that the hot molecular gas represents only a small fraction of the total amount of molecular gas, the estimated mass reservoir would be at least one order of magnitude larger than the mass needed to feed the AGN of NGC 4395 for an AGN cycle.

(vii) The FWHM of broad component of the $\mathrm{H} \alpha$ emission line is $\sim 785 \mathrm{~km} \mathrm{~s}^{-1}$ and the line-of-sight velocity of the BLR is blueshifted by $45 \mathrm{~km} \mathrm{~s}^{-1}$, relative to the narrow components. From the luminosity of the broad $\mathrm{H} \alpha$ component, we estimate a bolometric luminosity of $L_{\mathrm{bol}}=(9.9 \pm 1.4) \times 10^{40} \mathrm{erg} \mathrm{s}^{-1}$ for the AGN and estimate the mass of the SMBH as $M_{\mathrm{BH}}=\left(2.5_{-0.8}^{+1.0}\right) \times 10^{5} \mathrm{M}_{\odot}$, which is consistent with previous measurements.

NGC 4395 differs from typical Seyfert galaxies because (a) the mass inflow rate is lower, and (b) gas inflow in Seyferts tends to happen along nuclear dust spirals and bars, whereas NGC 4395 seems to be undergoing a minor merger or accretion event.

\section{ACKNOWLEDGEMENTS}

We thank the anonymous referee for valuable suggestions that helped to improve the paper. This work is based on observations obtained at the Gemini Observatory, which is operated by the Association of Universities for Research in Astronomy, Inc., under a cooperative agreement with the NSF on behalf of the Gemini partnership: the National Science Foundation (United States), the Science and Technology Facilities Council (United Kingdom), the National Research Council (Canada), CONICYT (Chile), the Australian Research Council (Australia), Ministério da Ciência e Tecnologia (Brazil), and south-east CYT (Argentina). CB, RAR, and RR acknowledge financial support from CNPq and FAPERGS. MRD thanks CAPES for financial support. The Brazilian authors thanks CAPES for financial support. LCH was supported by the
National Key R\&D Program of China (2016YFA0400702) and the National Science Foundation of China (11473002, 11721303).

\section{REFERENCES}

Abolfathi B. et al., 2018, ApJS, 235, 42

Akyuz A., Kayaci S., Avdan H., Ozel M. E., Sonbas E., Balman S., 2013, AJ, 145, 67

Allen M. G., Dopita M. A., Tsvetanov Z. I., 1998, ApJ, 493, 571

Baldwin J. A., Phillips M. M., Terlevich R., 1981, PASP, 93, 5

Belfiore F. et al., 2016, MNRAS, 461, 3111

Bertola F., Bettoni D., Danziger J., Sadler E., Sparke L., de Zeeuw T., 1991, ApJ, 373, 369

Brum C., Riffel R. A., Storchi-Bergmann T., Robinson A., Schnorr-Müller A., Lena D., 2017, MNRAS, 469, 3405

Cardelli J. A., Clayton G. C., Mathis J. S., 1989, ApJ, 345, 245

Carson D. J., Barth A. J., Seth A. C., den Brok M., Cappellari M., Greene J. E., Ho L. C., Neumayer N., 2015, AJ, 149, 170

Cedrés B., Cepa J., 2002, A\&A, 391, 809

Ceverino D., Sánchez Almeida J., Muh̆oz Tuhón C., Dekel A., Elmegreen B. G., Elmegreen D. M., Primack J., 2016, MNRAS, 457, 2605

Chilingarian I. V., Katkov I. Y., Zolotukhin I. Y., Grishin K. A., Beletsky Y., Boutsia K., Osip D. J., 2018, ApJ, 863, 1

Cid Fernandes R., Stasińska G., Schlickmann M. S., Mateus A., Vale Asari N., Schoenell W., Sodré L., Jr, 2010, MNRAS, 403, 1036

Cid Fernandes R., Stasińska G., Mateus A., Vale Asari N., 2011, MNRAS, 413,1687

Colina L. et al., 2015, A\&A, 578, A48

Cook D. O. et al., 2014, MNRAS, 445, 881

Couto G. S., Storchi-Bergmann T., Axon D. J., Robinson A., Kharb P., Riffel R. A., 2013, MNRAS, 435, 2982

Cresci G., Vanzi L., Telles E., Lanzuisi G., Brusa M., Mingozzi M., Sauvage M., Johnson K., 2017, A\&A, 604, A101

Dale D. A., Sheth K., Helou G., Regan M. W., Hüttemeister S., 2005, AJ, 129,2197

Davies R. I. et al., 2014, ApJ, 792, 101

Davies R. I., Müller Sánchez F., Genzel R., Tacconi L. J., Hicks E. K. S., Friedrich S., Sternberg A., 2007, ApJ, 671, 1388

den Brok M. et al., 2015, ApJ, 809, 101

Diniz M. R., Riffel R. A., Stochi-Bergmann T., Winge C., 2015, MNRAS, 453, 1727

Diniz M. R., Riffel R. A., Riffel R., Crenshaw D. M., Storchi-Bergmann T., Fischer T. C., Schmitt H. R., Kraemer S. B., 2017, MNRAS, 469, 3286

Dong X.-B., Ho L. C., Yuan W., Wang T.-G., Fan X., Zhou H., Jiang N., 2012, ApJ, 755, 167

Dors O. L., Cardaci M. V., Hägele G. F., Krabbe A. C., 2014, MNRAS, 443, 1291

Emsellem E., Krajnovic D., Sarzi M., 2014, MNRAS, 445, L79

Feltre A., Charlot S., Gutkin J., 2016, MNRAS, 456, 3354

Ferrarese L., Ford H., 2005, Space Sci. Rev., 116, 523

Ferrarese L., Merrit D., 2000, ApJ, 539, L9

Filippenko A. V., Ho L. C., 2003, ApJ, 588, L13

Filippenko A. V., Sargent W. L. W., 1989, ApJ, 342, L11

Filippenko A. V., Ho L. C., Sargent W. L. W., 1993, ApJ, 410, L75

Fischer T. C., Crenshaw D. M., Kraemer S. B., Schmitt H. R., StorchiBergmann T., Riffel R. A., 2015, ApJ, 799, 234

Frank J., , King A., Raine D. J., 2002, Accretion Power in Astrophysics, 3rd edn, Cambridge University Press.

Freitas I. C. et al., 2018, MNRAS, 476, 2760

Gebhardt K. et al., 2000, ApJ, 543, L5

Greene J. E., Ho L. C., 2004, ApJ, 610, 722

Greene J. E., Ho L. C., 2005, ApJ, 627, 721

Greene J. E., Ho L. C., 2005, ApJ, 630, 122

Greene J. E., Ho L. C., 2007, ApJ, 670, 92

Haynes M. P., Hogg D. E., Maddalena R. J., Roberts M. S., van Zee L., 1998, AJ, 115, 62

Hekatelyne C. et al., 2017, MNRAS, 474, 5319 
Hicks E. K. S., Davies R. I., Maciejewski W., Emsellem E., Malkan M. A., Dumas G., Müller-Sánchez F., Rivers A., 2013, ApJ, 768, 107

Ho L. C., 2009, ApJ, 699, 638

Ichikawa K., Ricci C., Ueda Y., Matsuoka K., Toba Y., Kawamuro T., Trakhtenbrot B., Koss M. J., 2017, ApJ, 835, 74

Jarrett T. H., Chester T., Cutri R., Schneider S. E., Huchra J. P., 2003, AJ, 125,525

Jeon M., Pawlik A. H., Greif T. H., Glover S. C. O., Bromm V., Milosavljević M., Klessen R. S., 2012, ApJ, 754, 34

Kakkad D. et al., 2018, A\&A, 618, A6

Kauffmann G. et al., 2003, MNRAS, 346, 1055

Kewley L. J., Heisler C. A., Dopita M. A., Lumsden S., 2001, ApJS, 132, 37

Kewley L. J., Groves B., Kauffmann G., Heckman T., 2006, MNRAS, 372, 961

Kishimoto M., Hönig S. F., Beckert T., Weigelt G., 2007, A\&A, 476, 713

Kong M., Ho L. C., 2018, ApJ, 859, 116

Kormendy J., Ho L. C., 2013, ARA\&A, 51, 511

Koski A. T., 1978, ApJ, 223, 56

Kraemer S. B., Ho L. C., Crenshaw D. M., Shields J. C., Filippenko A. V., 1999, ApJ, 520, 564

Kyuseok O. et al., 2018,

Lamperti I. et al., 2017, MNRAS, 467, 540

Larkin J. E., Armus L., Knop R. A., Soifer B. T., Matthews K., 1998, ApJS, 114,59

Lena D. et al., 2015, ApJ, 806, 84

Lena D., 2014, preprint (arXiv:1409.8264)

Lena D., Robinson A., Storchi-Bergmann T., Couto G. S., Schnorr-Müller A., Riffel R. A., 2016, MNRAS, 459, 4485

Ludwig R. R., Greene J. E., Barth A. J., Ho L. C., 2012, ApJ, 756, 51

Mallmann N. D. et al., 2018, MNRAS, 478, 5491

Markwardt C. B., 2009, in Bohlender D. A., Durand D., Dowler P., eds, ASP Conf. Ser., Vol. 411, Astronomical Data Analysis Software and Systems XVIII. Astron. Soc. Pac., San Francisco, p. 251

Martini P., Regan M. W., Mulchaey J. S., Pogge R. W., 2003, ApJS, 146, 353

Mazzalay X. et al., 2013, MNRAS, 428, 2389

McGregor P. J. et al., , 2003, in Masanori I., Moorwood A. F., eds, Proc. SPIE Conf. Ser., Vol. 4841, Instrument Design and Performance for Optical/Infrared Ground-based Telescopes. SPIE, Bellingham, p. 1581

Mezcua M., Kim M., Ho L. C., Lonsdale C. J., 2018, MNRAS, 480, L74

Moran E. C., Filippenko A.V., Ho L. C., Shields J. C., Belloni T., Comastri A., Snowden S. L., Sramek R. A., 1999, PASP, 111, 801

Mouri H., 1994, ApJ, 427, 777

Müller Sánchez F., Davies R. I., Eisenhauer F., Tacconi L. J., Genzel R., Sternberg A., 2006, A\&A, 454, 481

Müller Sánchez F., Davies R. I., Genzel R., Tacconi L. J., Eisenhauer F., Hicks E. K. S., Friedrich S., Sternberg A., 2009, ApJ, 691, 749

Müller-Sánchez F., Prieto M. A., Hicks E. K. S., Vives-Arias H., Davies R. I., Malkan M., Tacconi L. J., Genzel R., 2011, ApJ, 739, 69

Müller-Sánchez F., Nevin R., Comerford J. M., Davies R. I., Privon G. C., Treister E., 2018a, Nature, 556, 345

Müller-Sánchez F., Hicks E. K. S., Malkan M., Davies R., Yu P. C., Shaver S., Davis B., 2018b, ApJ, 858, 48

Oliva E. et al., 2001, A\&A, 369, L5

Osterbrock D. E., Ferland G. J., 2006, Astrophysics of Gaseous Nebulae and Active Galactic Nuclei, University Science Books, 2nd ed..

Peterson B. M. et al., 2003, ApJ, 632, 799

Raimundo S. I., Davies R. I., Gandhi P., Fabian A. C., Canning R. E. A., Ivanov V. D., 2013, MNRAS, 431, 2294

Raimundo S. I., Davies R. I., Canning R. E. A., Celotti A., Fabian A. C., Gandhi P., 2017, MNRAS, 464, 4227

Rayner J. T., Cushing M. C., Vacca W. D., 2009, ApJS, 185, 289
Reines A. E., Greene J. E., Geha M., 2013, ApJ, 775, 116

Reppeto R., Martínez-García E. E., Rosado M., Gabbasov R., 2017, MNRAS, 468, 180

Reunanen J., Kotilainen J. K., Prieto M. A., 2002, MNRAS, 331, 154

Riffel R. A. et al., 2015a, MNRAS, 446, 2823

Riffel R. et al., 2015b, MNRAS, 450, 3069

Riffel R. A., Storchi-Bergmann T., Riffel R., Dahmer- Hahn L. G., Diniz M. R., Schönell A. J., Dametto N. Z., 2017, MNRAS, 470, 992

Riffel R. A. et al., 2018, MNRAS, 474, 1373

Riffel R. A., 2010c, Ap\&SS, 327, 239

Riffel R. A., 2013, BAAA, 56, 13

Riffel R. A., Sorchi-Bergmann T., Winge C., Barbosa F. K. B., 2006a, MNRAS, 373, 2

Riffel R. A., Storchi-Bergmann T., Winge C., McGregor P. J., Beck T., Schmitt H., 2008, MNRAS, 385, 1129

Riffel R., Pastoriza M. G., Rodríguez-Ardila A., Bonatto C., 2008, MNRAS, 400, 273

Riffel R. A., Storchi-Bergmann T., Riffel R., Pastoriza M. G., 2010, ApJ, 713, 469

Riffel R. A., Storchi-Bergmann T., Nagar N. M., 2010, MNRAS, 404, 166

Riffel R., Riffel R. A., Ferrari F., Storchi-Bergmann T., 2011, MNRAS, 416, 493

Riffel R., Rodríguez-Ardila A., Aleman I., Brotherton M. S., Pastoriza M. G., Bonatto C., Dors O. L., 2013, MNRAS, 430, 2002

Riffel R. A., Storchi-Bergmann T., Winge C., 2013, MNRAS, 430, 2249

Riffel R. A., Vale T. B., Storchi-Bergmann T., McGregor P. J., 2014, MNRAS, 442, 656

Rodríguez-Ardila A., Pastoriza M. G., Viegas S., Sigut T. A. A., Pradhan A. K., 2004, A\&A, 425, 457

Rodríguez-Ardila A., Riffel R., Pastoriza M. G., 2005, MNRAS, 364, 1041

Rodríguez-Ardila A., Prieto M. A., Mazzalay X., Fernández-Ontiveros J. A., Luque R., Müller-Sánchez F., 2017, MNRAS, 470, 2845

Sánchez Almeida J. et al., 2015, ApJ, 810, L15

Sánchez S. F. et al., 2015, A\&A, 574, A47

Sarzi M. et al., 2010, MNRAS, 402, 2187

Schnorr Müller A., Storchi-Bergmann T., Nagar N. M., Ferrari F., 2014a, MNRAS, 438, 3322

Schnorr Müller A., Storchi-Bergmann T., Nagar N. M., Robinson A., Lena D., Riffel R. A., Couto G. S., 2014b, MNRAS, 437, 1708

Schönel A. J., Riffel R. A., Stochi-Bergmann T., Winge C., 2014, MNRAS, 445,414

Schönel A. J., Riffel R. A., Stochi-Bergmann T., Riffel R., 2017, MNRAS, 464,1771

Scoville N. Z., Hall D. N. B., Ridgway S. T., Kleinmann S. G., 1982, ApJ, 253,136

Shih D. C., Iwasawa K., Fabian A. C., 2003, MNRAS, 341, 973

Singh R. et al., 2013, A\&A, 558, A43

Stasińska G., Costa-Duarte M. V., Vale Asari N., Cid Fernandes R., Sodré L., 2015, MNRAS, 449, 559

Sternberg A., Dalgarno A., 1989, ApJ, 338, 197

Storchi-Bergmann T., McGregor P. J., Riffel R. A., Sim oes Lopes R., Beck T., Dopita M., 2009, MNRAS, 394, 1148

Tody D., 1986, in Crawford D. L., ed., Proc. SPIE Conf. Ser. Vol. 627, Instrumentation in Astronomy VI. SPIE, Bellingham, p. 733

Tody D., 1993, in Hanisch R. J., Brissenden R. J. V., Barnes J., eds, ASP Conf. Ser.Vol. 52, IRAF in the Nineties in Astronomical Data Analysis Software and Systems II. Astron. Soc. Pac., San Francisco, p. 173

Veilleux S., Osterbrock E. D., 1987, ApJ\&SS, 63, 295

Wrobel J. M., Ho L. C., 2006, ApJ, 646, L95

This paper has been typeset from a $\mathrm{T}_{\mathrm{E}} \mathrm{X} / \mathrm{L} \mathrm{T} \mathrm{E} \mathrm{X}$ file prepared by the author. 